\title{
Balanced solutions for an ellipsoidal vortex in a rotating stratified flow
}

\author{
By William J. McKiver ${ }^{1} \dagger$, and David G. Dritschel ${ }^{2}$ \\ ${ }^{1}$ ISMAR-CNR, Arsenale - Tesa 104, Castello 2737/F, 30122 Venice, Italy \\ ${ }^{2}$ School of Mathematics and Statistics, University of St Andrews, St Andrews KY16 9SS, UK
}

(Received ?; revised ?; accepted ?. - To be entered by editorial office)

We consider the motion of a single ellipsoidal vortex with uniform potential vorticity in a rotating stratified fluid at finite Rossby number $\epsilon$. Building on previous solutions obtained under the quasi-geostrophic approximation (at first order in $\epsilon$ ) we obtain analytical solutions for the balanced part of the flow at $\mathcal{O}\left(\epsilon^{2}\right)$. These solutions capture important ageostrophic effects giving rise to an asymmetry in the evolution of cyclonic and anti-cyclonic vortices.

Previous work has shown that if the velocity field induced by an ellipsoidal vortex only depends linearly on spatial coordinates inside the vortex, i.e. $\boldsymbol{u}=\mathbf{S x}$, then the dynamics reduces markedly to a simple matrix equation. The instantaneous vortex shape and orientation are encapsulated in a symmetric $3 \times 3$ matrix $\mathbf{B}$, which is acted upon by the flow matrix $\mathbf{S}$ to provide the vortex evolution. Under the quasi-geostrophic approximation, the flow matrix is determined by inverting the potential vorticity to obtain the streamfunction via Poisson's equation, which has a known analytical solution depending on elliptic integrals.

Here we show that higher-order balanced solutions, up to second order in the Rossby number, can also be calculated analytically. However, in this case there is a vector potential that requires the solution of three Poisson equations for each of its components. The source terms for these equations are independent of spatial coordinates within the ellipsoid, depending only on the elliptic integrals solved at the leading, quasi-geostrophic order. Unlike the quasi-geostrophic case these source terms do not in general vanish outside the ellipsoid and have an inordinately complicated dependence on spatial coordinates. In the special case of an ellipsoid whose axes are aligned with the coordinate axes, we are able to derive these source terms and obtain the full analytical solution to the three Poisson equations. However, if one considers the homogeneous case, whereby the outer source terms are neglected, one can obtain an approximate solution having a compact matrix form analogous to the leading-order quasi-geostrophic case. This approximate solution proves to be highly accurate for the general case of an arbitrarily-orientated ellipsoid, as verified through comparisons of the solutions with solutions obtained from numerical simulations of an ellipsoid using an accurate nonlinear balance model, even at moderate Rossby numbers.

\section{Introduction}

The study of vortices has long been considered fundamental to understanding the complex dynamics that underlie all turbulent fluid motions. This view arises naturally when

$\dagger$ Email address for correspondence: william.mckiver@ve.ismar.cnr.it 
one observes that turbulent flows contain an abundance of vortices, continually being generated, merging, breaking up, in a mêlée of complicated interactions. The nonlinear nature of these flows has meant that much of our understanding relies on numerical simulation. However, long before the advent of computer technology with the power to simulate very complicated turbulent fluid motions, already some of its underlying dynamics were elicited through the analysis of simple vortex models. This approach goes back as far as the work of Kirchhoff (1876), who showed that an isolated two-dimensional elliptical patch of uniform vorticity is an exact solution to the nonlinear Euler equations. Love (1893) followed this up by providing a stability analysis of this vortex. More recently works by Kida (1981) and Dritschel (1990) extended this model to the case when the elliptical patch is in the presence of uniform shearing and straining flows, where it was shown that the elliptical patch is broadly stable to non-elliptical disturbances in straining flows of practical interest.

Following on from this work the three-dimensional extension - to an ellipsoidal vortex — was developed by Meacham (1992) in the "quasi-geostrophic" limit, corresponding to a flow in rapid rotation and subject to strongly stable stratification (see McKiver (2015) for a recent review of the work on the quasi-geostrophic ellipsoid). Meacham considered this approach particularly relevant to deep, long-lived lens-like vortices formed in the Mediterranean, so-called "salt meddies". Observations have revealed that these vortices are characterised by a core of approximately homogeneous potential vorticity (D'Asaro (1988)), and have small Rossby numbers ( 0.1-0.2 Schultz-Tokos \& Rossby (1991)). Further studies by Meacham, Pankratov, Shchepetkin \& Zhmur (1994), Miyazaki, Ueno \& Shimonishi (1999), Hashimoto, Shimonishi \& Miyazaki (1999) and Miyazaki, Furuichi \& Takahashi (2001) examined different cases when there is an external straining flow. McKiver \& Dritschel (2003) introduced a new formulation that greatly simplified the equations, allowing one to solve the time evolution by means of a simple matrix equation and eigenvalue problem. This in turn led to a method for determining equilibria (Reinaud, Dritschel \& Koudella (2003)) and performing a full stability analysis of both the isolated vortex (Dritschel, Scott \& Reinaud (2005)) and the case when there is a background linear shear flow (McKiver \& Dritschel (2006)). Recent studies by Koshel et al. (2013, 2015) applied a slightly modified version of the ellipsoidal model in order to study how passive scalar transport through the vortex boundary is affected by advection and diffusion.

Recently a numerical study carried out by Tsang \& Dritschel (2015) considered the ellipsoidal vortex, beyond the quasi-geostrophic limit, in the full rotating stratified nonhydrostatic equations under the Boussinesq approximation. They examined the evolution of vortices having a range of different initial shapes at different values of the Rossby number up to unity in magnitude (cyclonic and anti-cyclonic) and determined "quasiequilibria". While exact equilibria do not exist because of the presence of inertia-gravity waves, they found that such waves are often exceedingly weak. When such waves can be neglected, the flow is said to be "balanced".

The concept of balance is based on the fact that in geophysical flows there exists two fundamentally different processes having very different timescales (Charney (1948); Warn, Bokhove, Shepherd \& Vallis (1995); Ford, McIntyre \& Norton (2000)). The first one arises from the relatively slow "balanced" motions driven primarily by potential vorticity (a materially-conserved scalar for an adiabatic, frictionless fluid), while the second one is the relatively fast oscillations due to inertia-gravity waves. When balance holds, inertia-gravity waves are neglected and the potential vorticity alone determines the evolution of the system. Over the years many balance models have been introduced, essentially trying to isolate more of the dynamics governed by the potential vorticity (Baer \& Tribbia (1977); Leith (1980); Vallis (1996); Bokhove (1997); Muraki, Snyder \& 
Rotunno (1999); Mohebalhojeh \& Dritschel (2001); Viúdez \& Dritschel (2004); McKiver \& Dritschel (2008)), and while a complete separation between the balanced dynamics and inertia-gravity waves appears to be impossible (Ford, McIntyre \& Norton (2000); Vanneste \& Yavneh (2004); Lane, Doyle, Plougonven, Shapiro \& Sharman (2004); Viúdez \& Dritschel (2006); Waite \& Bartello (2006)), many studies indicate that balance is still the dominant process up to Rossby numbers of order unity (Dritschel \& Viúdez (2007); McKiver \& Dritschel (2008); Dritschel \& McKiver (2015)).

Here we apply this concept of balance, specifically by using a balance model introduced by McKiver \& Dritschel (2008) for the non-hydrostatic Boussinesq equations, to solve for the motion of an ellipsoidal vortex at second order in Rossby number. We will exploit the matrix reformulation of the ellipsoidal dynamics introduced by McKiver \& Dritschel (2003), where the evolution of the ellipsoidal vortex depends on two $3 \times 3$ matrices. The first matrix, B, encapsulates the shape and orientation of the ellipsoid. The second matrix, $\mathbf{S}$, referred to as the "flow matrix", is related to the velocity field induced by the ellipsoid through $\boldsymbol{u}=\mathbf{S x}$. In fact, this linear dependence of the velocity field on spatial coordinates is necessary to preserve the ellipsoidal form of the vortex. That this is the case for the quasi-geostrophic (QG) ellipsoid has been known since Meacham (1992), though this does not hold at finite Rossby number within the full non-hydrostatic Boussinesq equations. However, we show that for a balance model at the next order beyond QG, one can find solutions that preserve this ellipsoidal form.

In section 2 we summarise the balance model used. We then recall the matrix formulation for the QG dynamics in section 3 (the leading-order solution in the balance model). In section 4 we derive balanced ellipsoidal solutions complete to second order in Rossby number. The analysis begins in section 4.1 by examining an approximation to the full equations where the source terms in the Poisson equations of the exterior potentials are set to zero (the "homogeneous solution" hereafter). In section 4.2 we consider two particular cases of steady (shape-invariant) motion and explicitly calculate the vortex rotation rate about the $z$-axis. For the first case in section 4.2.1, where the ellipsoidal axes are aligned with the coordinate axes, we derive the full analytical solution to the balance equations (including the external source terms) and compare it to the homogeneous solution as well as to numerical solutions of the nonlinear balance model. The second case considered in section 4.2.2 is that of a tilted spheroid for which we obtain the homogeneous solution and compare it with numerical solutions. In section 5 we draw our conclusions and suggest possible future avenues for exploration.

\section{Review of the nonlinear quasi-geostrophic balance model}

Here we review the derivation of the nonlinear quasi-geostrophic (NQG) balance model (McKiver \& Dritschel (2008)), which is based on a Rossby number expansion of the incompressible fluid dynamical equations under the Boussinesq approximation. The starting point is the reformulation introduced by Dritschel \& Viúdez (2003) that uses the potential vorticity (PV) alongside a pair of "thermal-wind imbalance" variables as the prognostic variables, rather than the standard velocity components and density. The first prognostic equation expresses the material conservation of $\mathrm{PV}$, or its anomaly $\varpi$ with respect to a constant background value:

$$
\frac{\mathrm{D} \varpi}{\mathrm{D} t} \equiv \varpi_{t}+\boldsymbol{u} \cdot \nabla \varpi=0
$$


Here a subscript $t$ denotes partial differentiation with respect to time, $\boldsymbol{u}=(u, v, w)$ is the velocity field, and the (dimensionless) PV anomaly $\varpi$ is defined as

$$
\varpi \equiv \frac{\zeta}{f}+\frac{b_{z}}{N^{2}}+\frac{\boldsymbol{\omega} \cdot \boldsymbol{\nabla} b}{f N^{2}}
$$

where $\boldsymbol{\omega}$ is the relative vorticity with $\zeta$ being its vertical component, $b$ is the buoyancy anomaly (see below), while $f$ and $N$ are the Coriolis and buoyancy frequencies respectively, both assumed to be constant. Throughout, subscripts $x, y$ and $z$ on fields denote partial differentiation. The total buoyancy is related to the density $\rho$ by $\bar{b}(z)+b(\boldsymbol{x}, t)=$ $-g\left(\rho-\rho_{0}\right) / \rho_{0}$, where $g$ is the acceleration due to gravity and $\rho_{0}$ is the mean density. The part depending only on $z$ is taken to be a linear function, $\bar{b}(z)=N^{2} z$, without loss of generality. The two other prognostic variables used are the horizontal components of the (dimensionless) ageostrophic vorticity, defined as

$$
\mathcal{A} \equiv(A, B, C)=\boldsymbol{\omega} / f+\nabla b / f^{2} .
$$

The horizontal components $(A, B)$ essentially express the departure from thermal-wind (geostrophic-hydrostatic) balance. Their time evolution is given by

$$
\begin{aligned}
& \frac{\mathrm{D} A}{\mathrm{D} t}=f B+\left(1-\chi^{2}\right) w_{x}+f^{-1} \boldsymbol{\omega} \cdot \boldsymbol{\nabla} u-f^{-2} \boldsymbol{u}_{x} \cdot \boldsymbol{\nabla} b, \\
& \frac{\mathrm{D} B}{\mathrm{D} t}=-f A+\left(1-\chi^{2}\right) w_{y}+f^{-1} \boldsymbol{\omega} \cdot \boldsymbol{\nabla} v-f^{-2} \boldsymbol{u}_{y} \cdot \boldsymbol{\nabla} b,
\end{aligned}
$$

where $\chi \equiv N / f$.

The NQG balance equations are derived from equations (2.2) and (2.4) by performing an expansion in the PV-based Rossby number, $\epsilon=|\varpi|_{\max }$, up to second order. The thermal-wind imbalance variables scale like $\epsilon^{2}$. Hence the horizontal components of the ageostrophic vorticity vanish at $\mathcal{O}(\epsilon)$ and only the linear terms remain in the definition of PV (2.2), i.e.

$$
\frac{\zeta^{1}}{f}+\frac{b_{z}^{1}}{N^{2}}=\varpi .
$$

Here and below, numerical superscripts on field variables only denote order in Rossby number. At $\mathcal{O}\left(\epsilon^{2}\right)$ quadratic terms appear in both (2.4) and (2.2) involving the first-order variables, i.e.

$$
\begin{aligned}
B^{2}+\frac{\left(1-\chi^{2}\right)}{f} w_{x}^{2} & =-\frac{1}{f^{2}}\left[\boldsymbol{\omega}^{1} \cdot \nabla u^{1}-\frac{1}{f} \boldsymbol{u}_{x}^{1} \cdot \nabla b^{1}\right], \\
A^{2}-\frac{\left(1-\chi^{2}\right)}{f} w_{y}^{2} & =\frac{1}{f^{2}}\left[\boldsymbol{\omega}^{1} \cdot \nabla v^{1}-\frac{1}{f} \boldsymbol{u}_{y}^{1} \cdot \nabla b^{1}\right], \\
\frac{\zeta^{2}}{f}+\frac{b_{z}^{2}}{N^{2}} & =-\frac{\boldsymbol{\omega}^{1} \cdot \boldsymbol{\nabla} b^{1}}{f N^{2}} .
\end{aligned}
$$

Expressing the velocity and buoyancy fields in terms of a vector potential, $\varphi \equiv(\varphi, \psi, \phi)$, one can obtain equations for $\varphi$ that can be inverted at each order. Here we write the velocity and buoyancy anomaly as

$$
\begin{aligned}
\boldsymbol{u} & =-f \boldsymbol{\nabla} \times \boldsymbol{\varphi}, \\
b & =N^{2}\left(\varphi_{x}+\psi_{y}\right)+f^{2} \phi_{z} .
\end{aligned}
$$

Now if we transform to a coordinate frame where the $z$ axis is stretched by $\chi \equiv N / f$ and substitute the vector potential $\varphi$ into the NQG equations (2.5) and (2.6), we obtain the 
QG equations at $\mathcal{O}(\epsilon)$

$$
\nabla^{2} \phi^{1}=\varpi
$$

and at $\mathcal{O}\left(\epsilon^{2}\right)$

$$
\begin{aligned}
\nabla^{2} \varphi^{2} & =\frac{2}{\chi}\left(\phi_{y y}^{1} \phi_{z x}^{1}-\phi_{y z}^{1} \phi_{x y}^{1}\right), \\
\nabla^{2} \psi^{2} & =\frac{2}{\chi}\left(\phi_{x x}^{1} \phi_{y z}^{1}-\phi_{z x}^{1} \phi_{x y}^{1}\right), \\
\nabla^{2} \phi^{2} & =\left|\nabla \phi_{z}^{1}\right|^{2}-\left(\nabla^{2} \phi^{1}\right) \phi_{z z}^{1} .
\end{aligned}
$$

The form of these equations are slightly different from those used in McKiver \& Dritschel (2008), because we have used a different expression for the buoyancy anomaly $b$ in terms of the vector potential components in (2.7), following Muraki, Snyder \& Rotunno (1999). This is deliberately done to obtain a more convenient inversion problem when dealing with the ellipsoidal vortex in the next section. The NQG balance model not only uses the above expanded equations for the second-order potentials, but also uses the exact definition of PV (equation 2.2) in an iterative loop to obtain a balanced solution (McKiver $\&$ Dritschel (2008)). In what follows we will derive analytical solutions to these equations for the case of the ellipsoid and will show that these solutions closely compare with the full iterative NQG balance solutions which use the exact definition of PV.

\section{Review of the quasi-geostrophic ellipsoidal vortex}

Next we review the solutions to the first-order part of the NQG equations, i.e. the QG equations, in the case of a single ellipsoid of uniform potential vorticity centred at the origin with axis half lengths $a \leqslant b \leqslant c$. The unit vectors $\hat{\mathbf{a}}, \hat{\mathbf{b}}$ and $\hat{\mathbf{c}}$ directed along these axes may be written

$$
\hat{\mathbf{a}}=\left(\begin{array}{c}
\hat{a}_{1} \\
\hat{a}_{2} \\
\hat{a}_{3}
\end{array}\right), \hat{\mathbf{b}}=\left(\begin{array}{c}
\hat{b}_{1} \\
\hat{b}_{2} \\
\hat{b}_{3}
\end{array}\right), \quad \hat{\mathbf{c}}=\left(\begin{array}{c}
\hat{c}_{1} \\
\hat{c}_{2} \\
\hat{c}_{3}
\end{array}\right) .
$$

Following the formulation of McKiver \& Dritschel (2003), the shape and orientation of the vortex is encapsulated in a single symmetric $3 \times 3$ matrix $\mathbf{B}$ defined by

$$
\mathbf{B}=a^{2} \hat{\mathbf{a}} \hat{\mathbf{a}}^{T}+b^{2} \hat{\mathbf{b}} \hat{\mathbf{b}}^{T}+c^{2} \hat{\mathbf{c}}^{T},
$$

where the superscript $T$ denotes transpose. From this matrix the axis lengths and orientation vectors can be determined from the solution of the eigenvalue problem:

$$
\begin{aligned}
\mathbf{B} \hat{\mathbf{a}} & =a^{2} \hat{\mathbf{a}}, \\
\mathbf{B} \hat{\mathbf{b}} & =b^{2} \hat{\mathbf{b}}, \\
\mathbf{B} \hat{\mathbf{c}} & =c^{2} \hat{\mathbf{c}} .
\end{aligned}
$$

If we assume that the velocity field in the vortex interior has the form

$$
\boldsymbol{u}=\mathbf{S} \boldsymbol{x},
$$

where $\mathbf{S}$ depends only on time $t$ (we refer to $\mathbf{S}$ as the flow matrix subsequently), then the equation of motion for the ellipsoid is simply

$$
\frac{d \mathbf{B}}{d t}=\mathbf{S B}+\mathbf{B S}^{T}
$$


(McKiver \& Dritschel (2003)). The elements of the flow matrix depend on the governing system of fluid dynamical equations. Notably, the only requirement is that the flow field within the ellipsoid has linear spatial dependence - this preserves the ellipsoidal form.

To obtain an ellipsoidal solution for the NQG equations, we need to show that the interior flow field is linear and calculate the flow matrix $\mathbf{S}$ to $\mathcal{O}\left(\epsilon^{2}\right)$. At first order in Rossby number $\epsilon$, the NQG balance equations derived in the previous section reduce to quasi-geostrophic balance (2.9), found simply by inverting

$$
\nabla^{2} \phi_{I}^{1}=Q_{\phi}^{1},
$$

for the interior vertical component of the potential $\phi_{I}^{1}$, where the source term is the (unexpanded) potential vorticity anomaly, i.e. $Q_{\phi}^{1}=\varpi$. The solution to this Poisson equation must match the solution $\phi_{E}^{1}$ external to the ellipsoid where the PV anomaly is zero, i.e.

$$
\nabla^{2} \phi_{E}^{1}=0
$$

These equations have a known analytical solution for the inner and outer fields. The inner field is given by (omitting the constant term)

$$
\phi_{I}^{1}=\frac{1}{2} \boldsymbol{x}^{T} \boldsymbol{\Phi}_{I}^{1} \boldsymbol{x},
$$

where $\Phi_{I}^{1}$ is the $3 \times 3$ symmetric matrix

$$
\boldsymbol{\Phi}_{I}^{1}=\xi_{a} \hat{\mathbf{a}} \hat{\mathbf{a}}^{T}+\xi_{b} \hat{\mathbf{b}} \hat{\mathbf{b}}^{T}+\xi_{c} \hat{\mathbf{c}} \hat{\mathbf{c}}^{T} .
$$

The coefficients $\xi_{a}, \xi_{b}$, and $\xi_{c}$ are given by

$$
\begin{aligned}
& \xi_{a}=\kappa_{v} R_{D}\left(b^{2}, c^{2}, a^{2}\right), \\
& \xi_{b}=\kappa_{v} R_{D}\left(c^{2}, a^{2}, b^{2}\right), \\
& \xi_{c}=\kappa_{v} R_{D}\left(a^{2}, b^{2}, c^{2}\right),
\end{aligned}
$$

where

$$
\kappa_{v}=\frac{\varpi a b c}{3}
$$

and where $R_{D}$ is the elliptic integral of the second kind defined by

$$
R_{D}(f, g, h) \equiv \frac{3}{2} \int_{0}^{\infty} \frac{d t}{\sqrt{(t+f)(t+g)(t+h)^{3}}} .
$$

The outer solution has the same form, i.e.

$$
\phi_{E}^{1}=\frac{1}{2} \boldsymbol{x}^{T} \boldsymbol{\Phi}_{E}^{1} \boldsymbol{x},
$$

but now the the matrix $\boldsymbol{\Phi}_{E}^{1}$ is given by

$$
\boldsymbol{\Phi}_{E}^{1}=\xi_{\alpha} \hat{\mathbf{a}} \hat{\mathbf{a}}^{T}+\xi_{\beta} \hat{\mathbf{b}} \hat{\mathbf{b}}^{T}+\xi_{\gamma} \hat{\mathbf{c}} \hat{\mathbf{c}}^{T},
$$

where

$$
\begin{aligned}
& \xi_{\alpha}=\kappa_{v} R_{D}\left(\beta^{2}, \gamma^{2}, \alpha^{2}\right), \\
& \xi_{\beta}=\kappa_{v} R_{D}\left(\gamma^{2}, \alpha^{2}, \beta^{2}\right), \\
& \xi_{\gamma}=\kappa_{v} R_{D}\left(\alpha^{2}, \beta^{2}, \gamma^{2}\right),
\end{aligned}
$$

and $\alpha^{2}=a^{2}+\lambda, \beta^{2}=b^{2}+\lambda, \gamma^{2}=c^{2}+\lambda$, with $\lambda$ being the largest root of the cubic equation

$$
\boldsymbol{x}^{T} \mathbf{A} \boldsymbol{x}=1
$$


where

$$
\mathbf{A}=\frac{\hat{\mathbf{a}} \hat{\mathbf{a}}^{T}}{a^{2}+\lambda}+\frac{\hat{\mathbf{b}} \hat{\mathbf{b}}^{T}}{b^{2}+\lambda}+\frac{\hat{\mathbf{c}} \hat{\mathbf{c}}^{T}}{c^{2}+\lambda}
$$

Note, $\lambda=0$ corresponds to the boundary of the ellipsoid. Since the velocity field at $\mathcal{O}(\epsilon)$ is given by $(u, v, w)=\left(-\partial \phi_{I}^{1} / \partial y, \partial \phi_{I}^{1} / \partial x, 0\right)$, it depends linearly on the spatial coordinates and so satisfies the evolution equation for the ellipsoid, equation (3.5). This solution for the uniform ellipsoid was in fact originally derived by Laplace (1784) for gravitating bodies with uniform mass. It was more recently applied by Meacham (1992) to the case of an ellipsoidal vortex in quasi-geostrophic flow. In general this solution applies when the Laplacian of some potential is equal to a uniform source term within the ellipsoid but a zero source term outside. When this is the case, the inner and outer solutions (the outer solution can be expanded in a series of ellipsoidal harmonics, see Appendix A) for the vertical potential and its gradient can be matched at the boundary, as was done for the QG ellipsoidal vortex by Meacham (1992). The solution derived by Laplace (1784) however is more general.

Given then the solution for the flow field, the flow matrix is given by

$$
\mathbf{S}^{1}=\mathbf{L}_{\phi} \boldsymbol{\Phi}_{I}^{1}
$$

where the skew matrix

$$
\mathbf{L}_{\phi}=\left(\begin{array}{ccc}
0 & -1 & 0 \\
1 & 0 & 0 \\
0 & 0 & 0
\end{array}\right)
$$

arises from the special form of the QG velocity field.

\section{Balanced ellipsoidal vortex}

\subsection{Homogeneous solution}

We next consider the NQG equations at $\mathcal{O}\left(\epsilon^{2}\right)$ for the ellipsoidal vortex. Unlike the case of the QG equations above, where there is a single streamfunction determining the flow field, the next order in NQG balance requires the full vector potential, $\varphi=(\varphi, \psi, \phi)$. If we substitute the first-order solutions $\phi^{1}$ in the equations at the next order, i.e. in equation (2.10), we can obtain the following equations for the interior potentials (after some manipulation):

$$
\begin{aligned}
\nabla^{2} \varphi_{I}^{2} & =Q_{\varphi}^{2}=-\frac{2}{\chi}\left(\xi_{b} \xi_{c} \hat{a}_{3} \hat{a}_{1}+\xi_{c} \xi_{a} \hat{b}_{3} \hat{b}_{1}+\xi_{a} \xi_{b} \hat{c}_{3} \hat{c}_{1}\right), \\
\nabla^{2} \psi_{I}^{2} & =Q_{\psi}^{2}=-\frac{2}{\chi}\left(\xi_{b} \xi_{c} \hat{a}_{2} \hat{a}_{3}+\xi_{c} \xi_{a} \hat{b}_{2} \hat{b}_{3}+\xi_{a} \xi_{b} \hat{c}_{2} \hat{c}_{3}\right), \\
\nabla^{2} \phi_{I}^{2} & =Q_{\phi}^{2}=-\xi_{a}\left(\xi_{b}+\xi_{c}\right) \hat{a}_{3}^{2}-\xi_{b}\left(\xi_{c}+\xi_{a}\right) \hat{b}_{3}^{2}-\xi_{c}\left(\xi_{a}+\xi_{b}\right) \hat{c}_{3}^{2} .
\end{aligned}
$$

The source terms on the right-hand sides, while complicated combinations of elliptic integrals and axes vector components, are spatially independent and at any instantaneous time they are uniform within the ellipsoidal vortex. However, unlike the case of the QG vortex, these source terms do not vanish outside the vortex. In fact, these source terms can be extremely complicated, mainly because of the dependence of the elliptic integrals coefficients, $\xi_{\alpha}, \xi_{\beta}$ and $\xi_{\gamma}$ on the spatial coordinates (see Appendix B and Appendix C).

In Appendix D we derive the exterior source terms for the special case of an ellipsoid in "standard position", where the coordinate axes are aligned with the axes of the ellipsoid (see Figure 1a). The full analytical solution in this case is presented in the next subsection. 
In the general case of an arbitrarily-orientated ellipsoid, however, the analysis appears to be prohibitively complicated due to the exceedingly complex forms of the exterior source terms. If one considers the case of a spherical vortex, i.e. $a=b=c$, the magnitude of these source terms can be estimated to be $\mathcal{O}\left(\varpi^{2} / r^{6}\right)$, where $r$ is the radial distance from the centre of the vortex. The small size of these terms together with their rapid decay may explain why, in practice, they contribute so weakly to the full solution (as shown below in section 4.2.2).

To make progress in the general case, we make an approximation which vastly simplifies the analysis: we simply neglect the exterior source terms. The accuracy of the homogeneous solution thereby obtained is tested in the next section, comparing it to both the full analytical solution as well as to the full iterative NQG balance solution (found numerically).

Setting the exterior source terms to zero allows us to apply directly Laplace's solution to the equations (4.1) to get

$$
\begin{aligned}
\varphi^{2} & =\frac{1}{2} \boldsymbol{x}^{T} \boldsymbol{\Gamma}_{I}^{2} \boldsymbol{x}, \\
\psi^{2} & =\frac{1}{2} \boldsymbol{x}^{T} \boldsymbol{\Psi}_{I}^{2} \boldsymbol{x}, \\
\phi^{2} & =\frac{1}{2} \boldsymbol{x}^{T} \boldsymbol{\Phi}_{I}^{2} \boldsymbol{x},
\end{aligned}
$$

where $\boldsymbol{\Gamma}_{I}^{2}, \boldsymbol{\Psi}_{I}^{2}$ and $\boldsymbol{\Phi}_{I}^{2}$ are $3 \times 3$ matrices given by

$$
\begin{aligned}
\boldsymbol{\Gamma}_{I}^{2} & =\frac{Q_{\varphi}^{2}}{\varpi} \boldsymbol{\Phi}^{1}, \\
\boldsymbol{\Psi}_{I}^{2} & =\frac{Q_{\psi}^{2}}{\varpi} \boldsymbol{\Phi}^{1}, \\
\boldsymbol{\Phi}_{I}^{2} & =\frac{Q_{\phi}^{2}}{\varpi} \boldsymbol{\Phi}^{1} .
\end{aligned}
$$

These solutions are second order in the PV anomaly $\varpi$ and hence in the Rossby number $\epsilon$. As the interior potentials have a quadratic dependence on spatial coordinates, the selfinduced velocity field is linear and preserves the ellipsoidal form. Thus, we can calculate the flow matrix using the definition of the velocity, equation (2.7), which can be written as

$$
\mathbf{S}^{2}=\mathbf{L}_{\varphi} \boldsymbol{\Gamma}_{I}^{2}+\mathbf{L}_{\psi} \mathbf{\Psi}_{I}^{2}+\mathbf{L}_{\phi} \boldsymbol{\Phi}_{I}^{2}
$$

where the skew matrices are defined as

$$
\mathbf{L}_{\varphi}=\left(\begin{array}{ccc}
0 & 0 & 0 \\
0 & 0 & -\chi \\
0 & \chi & 0
\end{array}\right), \quad \mathbf{L}_{\psi}=\left(\begin{array}{ccc}
0 & 0 & \chi \\
0 & 0 & 0 \\
-\chi & 0 & 0
\end{array}\right), \quad \mathbf{L}_{\phi}=\left(\begin{array}{ccc}
0 & -1 & 0 \\
1 & 0 & 0 \\
0 & 0 & 0
\end{array}\right)
$$

and where the scaling factor $\chi=N / f$ arises where the velocity field depends on $z$ derivatives of the vector potential components. Thus the general flow matrix up to $\mathcal{O}\left(\epsilon^{2}\right)$ can be written as

$$
\mathbf{S}=\frac{1}{\varpi}\left(Q_{\varphi} \mathbf{L}_{\varphi}+Q_{\psi} \mathbf{L}_{\psi}+Q_{\phi} \mathbf{L}_{\phi}\right) \Phi^{1}
$$

where the source terms $\left(Q_{\varphi}, Q_{\psi}, Q_{\phi}\right)$ are the sum of the first and second-order source terms defined in equations (3.6) and (4.1) respectively (note that at first order $Q_{\varphi}^{1}=$ $\left.Q_{\psi}^{1}=0\right)$. 
(a)

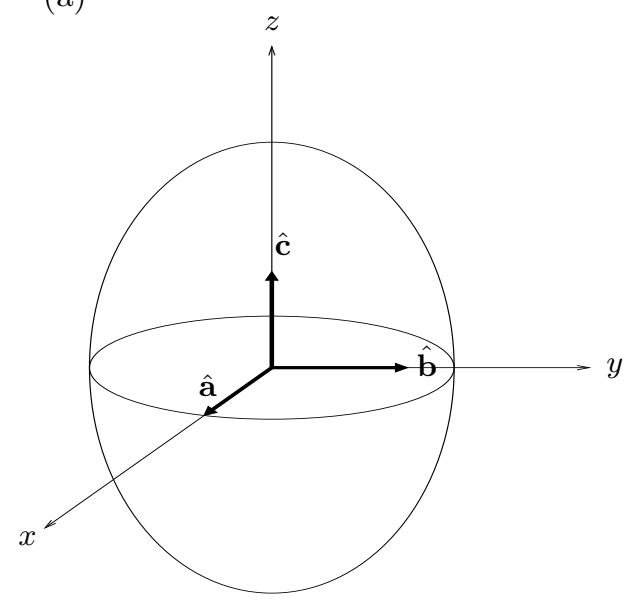

(b)

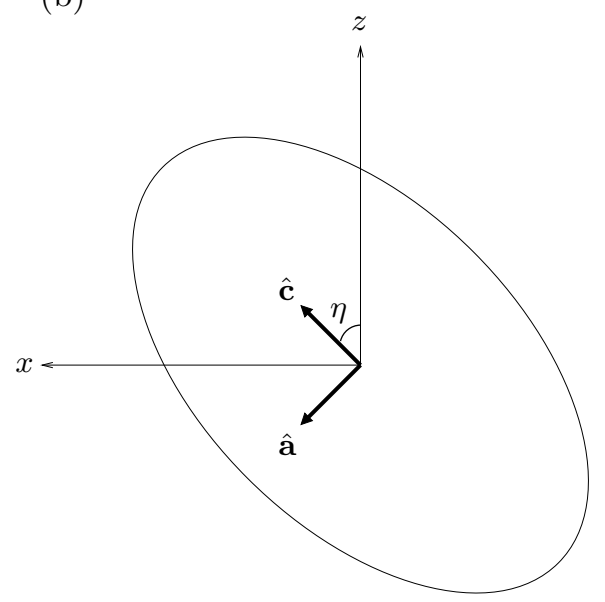

FiguRE 1. Schematic of the ellipsoidal vortex in two configurations: (a) standard position with the ellipsoidal axis $(\hat{\mathbf{a}}, \hat{\mathbf{b}}, \hat{\mathbf{c}})$ aligned with the coordinate axis $(x, y, z)$, (b) spheroidal vortex tilted by an angle $\eta$ about the $y$-axis.

\subsection{Particular solutions}

\subsubsection{The ellipsoid in standard position}

We now derive the complete analytical solution for an ellipsoid in standard position (see Figure 1a). In this case, the axes vectors are aligned with the Cartesian axes:

$$
\hat{\mathbf{a}}=\left(\begin{array}{l}
1 \\
0 \\
0
\end{array}\right), \quad \hat{\mathbf{b}}=\left(\begin{array}{l}
0 \\
1 \\
0
\end{array}\right), \quad \hat{\mathbf{c}}=\left(\begin{array}{l}
0 \\
0 \\
1
\end{array}\right) .
$$

In this case the solutions to the QG equations for the interior and exterior potentials (Laplace (1784); Meacham (1992)) are given by

$$
\begin{aligned}
\phi_{I}^{1} & =\frac{1}{2}\left(\xi_{a} x^{2}+\xi_{b} y^{2}+\xi_{c} z^{2}\right)-\frac{3}{2} \kappa_{v} R_{F}\left(a^{2}, b^{2}, c^{2}\right) \\
\phi_{E}^{1} & =\frac{1}{2}\left(\xi_{\alpha} x^{2}+\xi_{\beta} y^{2}+\xi_{\gamma} z^{2}\right)-\frac{3}{2} \kappa_{v} R_{F}\left(\alpha^{2}, \beta^{2}, \gamma^{2}\right)
\end{aligned}
$$

where $R_{F}$ is the elliptic integral of the first kind:

$$
R_{F}(x, y, z) \equiv \frac{1}{2} \int_{0}^{\infty} \frac{d t}{\sqrt{(t+x)(t+y)(t+z)}}
$$

(note $R_{F}$ is equivalent to the first-order Lamé function of the second kind, $\mathbf{F}_{0}^{(1)}$, see Appendix A). If we substitute the first-order (QG) solutions $\phi^{1}$ in the equations at the next order, i.e. in equation (2.10), we obtain the following equations for the interior potentials:

$$
\begin{aligned}
\nabla^{2} \varphi_{I}^{2} & =0 \\
\nabla^{2} \psi_{I}^{2} & =0 \\
\nabla^{2} \phi_{I}^{2} & =-\xi_{c}\left(\xi_{a}+\xi_{b}\right) .
\end{aligned}
$$


For the exterior potentials (see Appendix D for derivation) we have

$$
\begin{aligned}
& \nabla^{2} \varphi_{E}^{2}=-\left(\frac{6 \kappa_{v} \alpha^{2} \beta^{4} \gamma^{2} \xi_{\beta}}{\chi \Delta_{0} K\left(\alpha^{4}, \beta^{4}, \gamma^{4}\right)}\right) z x \\
& \nabla^{2} \psi_{E}^{2}=-\left(\frac{6 \kappa_{v} \alpha^{4} \beta^{2} \gamma^{2} \xi_{\alpha}}{\chi \Delta_{0} K\left(\alpha^{4}, \beta^{4}, \gamma^{4}\right)}\right) y z, \\
& \nabla^{2} \phi_{E}^{2}=\xi_{\gamma}^{2}+\left[\frac{3 \kappa_{v} \alpha^{4} \beta^{4}}{\Delta_{0} K\left(\alpha^{4}, \beta^{4}, \gamma^{4}\right)}\left(\frac{3 \kappa_{v}}{\Delta_{0}}-2 \xi_{\gamma}\right)\right] z^{2},
\end{aligned}
$$

where $\Delta_{0}$ and the function $K\left(\alpha^{4}, \beta^{4}, \gamma^{4}\right)$ are defined in equations (C.6) and (B.4) respectively. We impose continuity of velocity, buoyancy and pressure at the ellipsoidal boundary; this is equivalent to imposing continuity of the potentials and their first 'radial' derivative, i.e.

$$
\begin{aligned}
\left.\boldsymbol{\varphi}_{I}\right|_{\lambda=0} & =\left.\boldsymbol{\varphi}_{E}\right|_{\lambda=0}, \\
\left.\frac{\partial \varphi_{I}}{\partial \lambda}\right|_{\lambda=0} & =\left.\frac{\partial \varphi_{E}}{\partial \lambda}\right|_{\lambda=0}
\end{aligned}
$$

where $\lambda$ is a coordinate analogous to the radial coordinate in spherical coordinates. In particular, surfaces of constant $\lambda$ correspond to the ellipsoids

$$
\frac{x^{2}}{a^{2}+\lambda}+\frac{y^{2}}{b^{2}+\lambda}+\frac{z^{2}}{c^{2}+\lambda}=1 .
$$

Given the form of the source terms in the Poisson equations, we seek solutions for the interior potentials of the form

$$
\begin{aligned}
\varphi_{I}^{2} & =A_{I} z x, \\
\psi_{I}^{2} & =B_{I} y z \\
\phi_{I}^{2} & ==\frac{1}{2}\left(D_{I} x^{2}+E_{I} y^{2}+F_{I} z^{2}\right)+C_{I}
\end{aligned}
$$

where the coefficients $A_{I}, B_{I}, C_{I}, D_{I}, E_{I}$ and $F_{I}$ are constants. Regarding the form of the exterior potentials, we split the solution for each into a particular solution, $P$, satisfying its Poisson equation, and a homogeneous solution composed of ellipsoidal harmonics, $H$, in order to satisfy the boundary conditions. It can be shown by direct substitution into equation (4.11) and using the Laplacian formula (B.13) that the particular solutions needed to satisfy the Poisson equations (4.11) are

$$
\begin{aligned}
P_{\varphi} & =-\frac{1}{\chi} \xi_{\gamma} \xi_{\alpha} z x, \\
P_{\psi} & =-\frac{1}{\chi} \xi_{\beta} \xi_{\gamma} y z, \\
P_{\phi} & =\frac{1}{2} \xi_{\gamma}^{2} z^{2} .
\end{aligned}
$$

For the homogeneous part, we look for solutions in ellipsoidal harmonics (see Appendix A), as was the approach of Meacham (1992). Given the quadratic form of the inner solutions, the appropriate choice needed in order to match the solutions at the boundary are

$$
\begin{aligned}
& H_{\varphi}=A_{E} \mathbf{F}_{2}^{(4)}(\lambda) \mathbf{E}_{2}^{(4)}(\mu) \mathbf{E}_{2}^{(4)}(\nu) \\
& H_{\psi}=B_{E} \mathbf{F}_{2}^{(3)}(\lambda) \mathbf{E}_{2}^{(3)}(\mu) \mathbf{E}_{2}^{(3)}(\nu) \\
& H_{\phi}=C_{E} \mathbf{F}_{0}^{(1)}(\lambda)+D_{E} \mathbf{F}_{2}^{(1)}(\lambda) \mathbf{E}_{2}^{(1)}(\mu) \mathbf{E}_{2}^{(1)}(\nu)+E_{E} \mathbf{F}_{2}^{(2)}(\lambda) \mathbf{E}_{2}^{(2)}(\mu) \mathbf{E}_{2}^{(2)}(\nu)
\end{aligned}
$$


where the coefficients $A_{E}, B_{E}, C_{E}, D_{E}$ and $E_{E}$ are constants. The interior and exterior coefficients can be solved by writing the equations in ellipsoidal coordinates using equation (A.1), and then applying the boundary conditions, equation (4.12). Beginning with the $\varphi$ potential, the inner and outer potentials in ellipsoidal coordinates are

$$
\begin{aligned}
\varphi_{I}^{2} & =\frac{\gamma \alpha A_{I} \mathbf{E}_{2}^{(4)}(\mu) \mathbf{E}_{2}^{(4)}(\nu)}{\left(c^{2}-a^{2}\right) \sqrt{\left(b^{2}-c^{2}\right)\left(a^{2}-b^{2}\right)}} \\
\varphi_{E}^{2} & =\left[A_{E} \mathbf{F}_{2}^{(4)}(\lambda)-\frac{\xi_{\gamma} \xi_{\alpha} \gamma \alpha}{\chi\left(c^{2}-a^{2}\right) \sqrt{\left(b^{2}-c^{2}\right)\left(a^{2}-b^{2}\right)}}\right] \mathbf{E}_{2}^{(4)}(\mu) \mathbf{E}_{2}^{(4)}(\nu),
\end{aligned}
$$

and their derivatives are

$$
\begin{aligned}
\frac{\partial \varphi_{I}^{2}}{\partial \lambda} & =\frac{A_{I} \gamma \alpha \mathbf{E}_{2}^{(4)}(\mu) \mathbf{E}_{2}^{(4)}(\nu)}{\left(c^{2}-a^{2}\right) \sqrt{\left(b^{2}-c^{2}\right)\left(a^{2}-b^{2}\right)}} \\
\frac{\partial \varphi_{E}^{2}}{\partial \lambda} & =\left[A_{E} \frac{d \mathbf{F}_{2}^{(4)}}{d \lambda}+\frac{3 \kappa_{v}\left(\gamma^{2} \xi_{\gamma}+\alpha^{2} \xi_{\alpha}\right)-\xi_{\gamma} \xi_{\alpha}\left(\gamma^{2}+\alpha^{2}\right) \Delta_{0}}{2 \chi \gamma \alpha \Delta_{0}\left(c^{2}-a^{2}\right) \sqrt{\left(b^{2}-c^{2}\right)\left(a^{2}-b^{2}\right)}}\right] \mathbf{E}_{2}^{(4)}(\mu) \mathbf{E}_{2}^{(4)}(\nu) .
\end{aligned}
$$

Matching the interior and exterior potentials at the boundary $(\lambda=0)$ provides two equations in the two unknown coefficients that we can solve for, giving

$$
\begin{aligned}
A_{I} & =\frac{a^{2} \xi_{a}^{2}-c^{2} \xi_{c}^{2}}{\chi\left(c^{2}-a^{2}\right)} \\
A_{E} & =\frac{3 \kappa_{v}\left(c^{2} \xi_{c}+a^{2} \xi_{a}\right)}{5 \chi\left(c^{2}-a^{2}\right) \sqrt{\left(b^{2}-c^{2}\right)\left(a^{2}-b^{2}\right)}}
\end{aligned}
$$

Similarly, we obtain the $\psi$ coefficients:

$$
\begin{aligned}
B_{I} & =\frac{c^{2} \xi_{c}^{2}-b^{2} \xi_{b}^{2}}{\chi\left(b^{2}-c^{2}\right)} \\
B_{E} & =\frac{3 \kappa_{v}\left(b^{2} \xi_{b}+c^{2} \xi_{c}\right)}{5 \chi\left(b^{2}-c^{2}\right) \sqrt{\left(a^{2}-b^{2}\right)\left(c^{2}-a^{2}\right)}}
\end{aligned}
$$

For the $\phi$ potential, when transformed to ellipsoidal coordinates we can write the interior and exterior solutions as

$$
\begin{aligned}
\phi_{I}^{2} & =\mu \nu\left[D_{I} \alpha^{2}\left(b^{2}-c^{2}\right)+E_{I} \beta^{2}\left(c^{2}-a^{2}\right)+F_{I} \gamma^{2}\left(a^{2}-b^{2}\right)\right] M \\
& +(\mu+\nu)\left[D_{I} \alpha^{2} a^{2}\left(b^{2}-c^{2}\right)+E_{I} \beta^{2} b^{2}\left(c^{2}-a^{2}\right)+F_{I} \gamma^{2} c^{2}\left(a^{2}-b^{2}\right)\right] M \\
& +\left[D_{I} \alpha^{2} a^{4}\left(b^{2}-c^{2}\right)+E_{I} \beta^{2} b^{4}\left(c^{2}-a^{2}\right)+F_{I} \gamma^{2} c^{4}\left(a^{2}-b^{2}\right)\right] M+C_{I} \\
\phi_{E}^{2} & =\mu \nu\left[D_{E} \mathbf{F}_{2}^{(1)}(\lambda)+E_{E} \mathbf{F}_{2}^{(2)}(\lambda)+\xi_{\gamma}^{2} \gamma^{2}\left(a^{2}-b^{2}\right) M\right] \\
& +(\mu+\nu)\left[D_{E} d_{1} \mathbf{F}_{2}^{(1)}(\lambda)+E_{E} d_{2} \mathbf{F}_{2}^{(2)}(\lambda)+\xi_{\gamma}^{2} \gamma^{2} c^{2}\left(a^{2}-b^{2}\right) M\right] \\
& +C_{E} \mathbf{F}_{0}^{(1)}(\lambda)+D_{E} d_{1}^{2} \mathbf{F}_{2}^{(1)}(\lambda)+E_{E} d_{2}^{2} \mathbf{F}_{2}^{(2)}(\lambda)+\xi_{\gamma}^{2} \gamma^{2} c^{4}\left(a^{2}-b^{2}\right) M,
\end{aligned}
$$


and their derivatives are

$$
\begin{aligned}
\frac{\partial \phi_{I}^{2}}{\partial \lambda} & =\mu \nu\left[D_{I}\left(b^{2}-c^{2}\right)+E_{I}\left(c^{2}-a^{2}\right)+F_{I}\left(a^{2}-b^{2}\right)\right] M \\
& +(\mu+\nu)\left[D_{I} a^{2}\left(b^{2}-c^{2}\right)+E_{I} b^{2}\left(c^{2}-a^{2}\right)+F_{I} c^{2}\left(a^{2}-b^{2}\right)\right] M \\
& +\left[D_{I} a^{4}\left(b^{2}-c^{2}\right)+E_{I} b^{4}\left(c^{2}-a^{2}\right)+F_{I} c^{4}\left(a^{2}-b^{2}\right)\right] M \\
\frac{\partial \phi_{E}^{2}}{\partial \lambda} & =\mu \nu\left[D_{E} \frac{d \mathbf{F}_{2}^{(1)}}{d \lambda}+E_{E} \frac{d \mathbf{F}_{2}^{(2)}}{d \lambda}-\xi_{\gamma}\left(\xi_{\alpha}+\xi_{\beta}\right)\left(a^{2}-b^{2}\right) M\right] \\
& +(\mu+\nu)\left[D_{E} d_{1} \frac{d \mathbf{F}_{2}^{(1)}}{d \lambda}+E_{E} d_{2} \frac{d \mathbf{F}_{2}^{(2)}}{d \lambda}-\xi_{\gamma}\left(\xi_{\alpha}+\xi_{\beta}\right) c^{2}\left(a^{2}-b^{2}\right) M\right] \\
& +C_{E} \frac{d \mathbf{F}_{0}^{(1)}}{d \lambda}+D_{E} d_{1}^{2} \frac{d \mathbf{F}_{2}^{(1)}}{d \lambda}+E_{E} d_{2}^{2} \frac{d \mathbf{F}_{2}^{(2)}}{d \lambda}-\xi_{\gamma}\left(\xi_{\alpha}+\xi_{\beta}\right) c^{4}\left(a^{2}-b^{2}\right) M
\end{aligned}
$$

where $d_{1}$ and $d_{2}$ are constants from the Lamé functions defined in equations (A.6a,b) respectively, and where

$$
M \equiv-\frac{1}{2\left(a^{2}-b^{2}\right)\left(c^{2}-a^{2}\right)\left(b^{2}-c^{2}\right)} .
$$

Matching the interior and exterior potentials and their derivatives at the boundary $(\lambda=$ 0 ) provide 6 conditions, since there are terms proportional to $\mu \nu$ and $\mu+\nu$ as well as the constant terms. Another equation is provided by substituting equation (4.14c) into equation (4.10c) giving

$$
D_{I}+E_{I}+F_{I}=-\xi_{c}\left(\xi_{a}+\xi_{b}\right)
$$

Thus we have 7 equations in the 7 unknown coefficients which can be solved giving

$$
\begin{aligned}
C_{I} & =\frac{1}{2} c^{2} \xi_{c}^{2} \\
D_{I} & =\frac{c^{2} \xi_{c}\left(\xi_{c}-\xi_{a}\right)}{c^{2}-a^{2}} \\
E_{I} & =\frac{c^{2} \xi_{c}\left(\xi_{b}-\xi_{c}\right)}{b^{2}-c^{2}} \\
F_{I} & =\xi_{c}\left(\frac{a^{2} \xi_{a}-c^{2} \xi_{c}}{c^{2}-a^{2}}+\frac{c^{2} \xi_{c}-b^{2} \xi_{b}}{b^{2}-c^{2}}\right) \\
C_{E} & =0 \\
D_{E} & =\frac{6 \xi_{c} \kappa_{v}\left(d_{2}-c^{2}\right)\left(a^{2}-b^{2}\right) M c^{2}}{5\left(d_{1}-d_{2}\right)} \\
E_{E} & =-\frac{6 \xi_{c} \kappa_{v}\left(d_{1}-c^{2}\right)\left(a^{2}-b^{2}\right) M c^{2}}{5\left(d_{1}-d_{2}\right)}
\end{aligned}
$$

Now the solution for the special case of the ellipsoid in standard position for the NQG system can be written as

$$
\begin{aligned}
\varphi_{I}^{2} & =\frac{1}{2} \boldsymbol{x}^{T} \boldsymbol{\Gamma}_{I}^{2} \boldsymbol{x}, \\
\psi_{I}^{2} & =\frac{1}{2} \boldsymbol{x}^{T} \boldsymbol{\Psi}_{I}^{2} \boldsymbol{x}, \\
\phi_{I}^{2} & =\frac{1}{2} \boldsymbol{x}^{T} \boldsymbol{\Phi}_{I}^{2} \boldsymbol{x},
\end{aligned}
$$


where the $3 \times 3$ matrices $\boldsymbol{\Gamma}^{2}, \boldsymbol{\Psi}^{2}$ and $\boldsymbol{\Phi}^{2}$ are

$$
\boldsymbol{\Gamma}^{2}=\left(\begin{array}{ccc}
0 & 0 & A_{I} \\
0 & 0 & 0 \\
A_{I} & 0 & 0
\end{array}\right), \quad \boldsymbol{\Psi}^{2}=\left(\begin{array}{ccc}
0 & 0 & 0 \\
0 & 0 & B_{I} \\
0 & B_{I} & 0
\end{array}\right), \quad \boldsymbol{\Phi}^{2}=\left(\begin{array}{ccc}
D_{I} & 0 & 0 \\
0 & E_{I} & 0 \\
0 & 0 & F_{I}
\end{array}\right) \text {. }
$$

Note the exterior solutions have the same form. The flow matrix is calculated using equation (4.4), and is given by

$$
\mathbf{S}=\left(\begin{array}{ccc}
0 & -\xi_{b}+\chi B_{I}-E_{I} & 0 \\
\xi_{a}+D_{I}-\chi A_{I} & 0 & 0 \\
0 & 0 & 0
\end{array}\right)
$$

The form of the flow matrix indicates that the only time-dependent part of the $\mathbf{B}$ matrix is the $\mathbf{B}_{12}=\mathbf{B}_{21}$ element, implying constant rotation about the $z$ axis. If we shift to a reference frame that is rotating about the $z$-axis with the ellipsoid then we can write

$$
\left(\mathbf{S}-\Omega \mathbf{L}_{\phi}\right) \mathbf{B}+\mathbf{B}\left(\mathbf{S}-\Omega \mathbf{L}_{\phi}\right)^{T}=0,
$$

where $\Omega$ is the rotation rate about the $z$-axis and $\mathbf{L}_{\phi}$ is the skew matrix (equation (3.19)). As the ellipsoid's axes are aligned with the coordinate axes, the matrix $\mathbf{B}$ reduces to a diagonal matrix with elements $a^{2}, b^{2}$ and $c^{2}$. Substituting for the above flow matrix into equation (4.29) we can solve for the rotation rate

$$
\Omega=\Omega^{(1)}+\Omega^{(2)}
$$

where $\Omega^{(1)}$ is the $\mathrm{QG}$ rotation rate given by

$$
\Omega^{(1)}=\frac{a^{2} \xi_{a}-b^{2} \xi_{b}}{a^{2}-b^{2}}
$$

(Meacham (1992), Dritschel, Scott \& Reinaud (2005)). The second term, $\Omega^{(2)}$, is the correction for the balanced solution at the next order. It is given by

$$
\Omega^{(2)}=a^{2}\left[\frac{2 c^{2} \xi_{c}^{2}-a^{2} \xi_{a}^{2}-c^{2} \xi_{c} \xi_{a}}{\left(a^{2}-b^{2}\right)\left(c^{2}-a^{2}\right)}\right]+b^{2}\left[\frac{2 c^{2} \xi_{c}^{2}-b^{2} \xi_{b}^{2}-c^{2} \xi_{b} \xi_{c}}{\left(b^{2}-c^{2}\right)\left(a^{2}-b^{2}\right)}\right]
$$

We can also write the second-order solution based on the approximate homogeneous solution given by equation $(4.3 \mathrm{c})$. This provides a simple formula for the rotation rate, denoted $\Omega_{H}^{(2)}$, i.e.

$$
\Omega_{H}^{(2)}=-\frac{\Omega^{(1)} \xi_{c}}{\varpi}\left(\xi_{a}+\xi_{b}\right) .
$$

We now have two different analytical solutions based on the NQG equations up to second order in the Rossby number, the first being the complete solution while the second one is based on a simplified form of the balance equations. However, neither of these analytical solutions use directly the full definition of PV. The use of the unapproximated form of PV through the inversion of the full equations has been shown to be of great importance for capturing the balanced component of the flow dynamics (Ford, McIntyre \& Norton (2000); Mohebalhojeh (2002); McKiver \& Dritschel (2008)). In order to check the level of balance captured by the analytical solutions, we compare the analytical rotation rates with that computed numerically using the full NQG model. The numerical scheme used defines the ellipsoid of PV on a grid and solves directly for the NQG streamfunction through an iterative inversion of the full nonlinear Monge-Ampère equation, and using pseudo spectral methods to apply derivatives of the various potentials (see Tsang \& Dritschel (2015) for full details of this numerical scheme). The grid is a triply periodic 
14

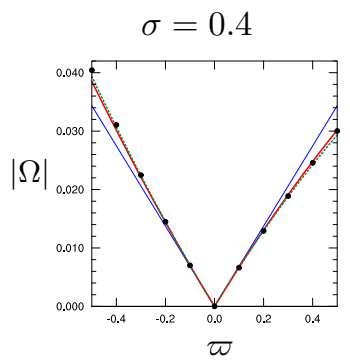

W. J. McKiver and D. G. Dritschel

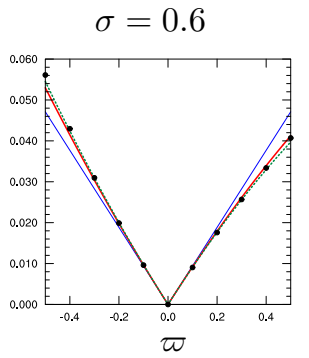

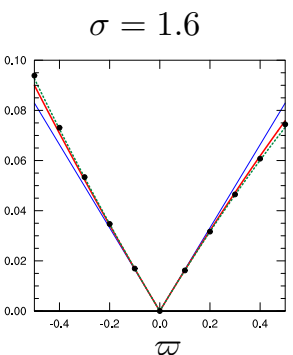

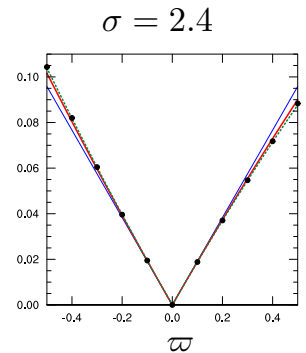

Figure 2. Plots of $|\Omega|$ versus $\varpi$ for an ellipsoid in standard position for four different values of the vertical aspect ratio $\sigma=c / \sqrt{a b}$, all for a fixed horizontal aspect ratio of $\delta=a / b=0.8$. The thin blue curve is the QG rotation rate $\left|\Omega^{(1)}\right|$, the thick red curve is the homogeneous balance rotation rate $|\Omega|=\left|\Omega^{(1)}+\Omega_{H}^{(2)}\right|$, the green dashed curve is the complete analytical solution $|\Omega|=\left|\Omega^{(1)}+\Omega^{(2)}\right|$ and the black points are the NQG balance values obtained numerically.

domain with a grid size of $256^{3}$. The domain dimensions are $(2 \pi)^{3}$ with the mean radius of the vortex chosen to be $(a b c)^{1 / 3}=0.5$ so as to reduce the effects of periodicity. As this scheme uses the unapproximated form of PV it is extremely accurate in capturing the balanced component of the flow (McKiver \& Dritschel (2008); Dritschel \& McKiver (2015)). This numerical method was run for a range of different values of the horizontal aspect ratio, $\delta \equiv a / b$, the vertical aspect ratio $\sigma=c / \sqrt{a b}$, and the anomalous PV, $\varpi$.

For an ellipsoid in standard position, figure 2 compares the numerically-computed rotation rate with the $\mathrm{QG}$ approximation as well as the two estimates at next order in Rossby number. Here, we take a horizontal aspect ratio $\delta=0.8$, and four different values of the vertical aspect ratio, $\sigma$ (these are representative). The QG solution has some skill in capturing the rotation rate at lower Rossby numbers $(|\varpi|<0.2)$, but as the PV increases, a noticeable asymmetry appears in the rotation rates for cyclonic and anti-cyclonic vortices. This departure from the QG solution is well captured by both the analytical solutions for the NQG. The only discrepancy occurs for strongly anti-cyclonic vortices, which are known to be significantly more ageostrophic than their cyclonic counterparts (Tsang \& Dritschel (2015)). The two analytical solutions themselves are almost indistinguishable, with only slight differences appearing at the highest Rossby number where the complete analytical solution gives a better estimate than the homogeneous solution in the case of a strongly anti-cyclonic vortex. The asymmetric behaviour of the cyclonic and anti-cyclonic rotation rates computed here has also been noted in a previous study by Schecter \& Montgomery (2003). That study also derived analytical solutions for the ageostrophic correction to QG, albeit only for a Rankine (barotropic) vortex surrounded by a skirt of low-lying vorticity.

\subsubsection{Tilted spheroid}

We consider next the case of a spheroidal vortex $(a=b)$ tilted about the $y$-axis by an angle $\eta$ with respect to the $z$-axis (see Figure 1b). Like the case of the ellipsoid in standard position it has been shown by Miyazaki, Ueno \& Shimonishi (1999) that, under the QG approximation, a spheroidal vortex also rotates steadily about the $z$-axis. For this case we do not solve the full potential problem, but only the homogeneous case where the exterior source terms are set to zero. We start by first considering the general tilted ellipsoid for which the vortex axis vectors are

$$
\hat{\mathbf{a}}=\left(\begin{array}{c}
\cos \eta \\
0 \\
-\sin \eta
\end{array}\right), \quad \hat{\mathbf{b}}=\left(\begin{array}{l}
0 \\
1 \\
0
\end{array}\right), \quad \hat{\mathbf{c}}=\left(\begin{array}{c}
\sin \eta \\
0 \\
\cos \eta
\end{array}\right) .
$$


and for which the elements of the symmetric matrix $\mathbf{B}$ are

$$
\begin{aligned}
& \mathbf{B}_{11}=a^{2}+\left(c^{2}-a^{2}\right) \sin ^{2} \eta ; \quad \mathbf{B}_{22}=b^{2} ; \quad \mathbf{B}_{33}=c^{2}-\left(c^{2}-a^{2}\right) \sin ^{2} \eta ; \\
& \mathbf{B}_{31}=B_{13}=\left(c^{2}-a^{2}\right) \sin \eta \cos \eta ; \quad \mathbf{B}_{12}=\mathbf{B}_{23}=0 .
\end{aligned}
$$

The leading-order QG solution of Poisson's equation (3.8) is written in terms of the symmetric matrix $\boldsymbol{\Phi}^{1}$ (equation (3.9)) and has components

$$
\begin{aligned}
& \boldsymbol{\Phi}_{11}^{1}=\xi_{a}+\left(\xi_{c}-\xi_{a}\right) \sin ^{2} \eta ; \quad \boldsymbol{\Phi}_{22}^{1}=\xi_{a} ; \quad \boldsymbol{\Phi}_{33}^{1}=\xi_{c}-\left(\xi_{c}-\xi_{a}\right) \sin ^{2} \eta ; \\
& \boldsymbol{\Phi}_{31}^{1}=\boldsymbol{\Phi}_{13}^{1}=\left(\xi_{c}-\xi_{a}\right) \sin \eta \cos \eta ; \quad \boldsymbol{\Phi}_{12}^{1}=\boldsymbol{\Phi}_{23}^{1}=0 .
\end{aligned}
$$

The interior source terms needed for the next-order solutions are

$$
\begin{aligned}
Q_{\varphi}^{2} & =2 \xi_{a}\left(\xi_{c}-\xi_{a}\right) \sin \eta \cos \eta \\
Q_{\psi}^{2} & =0 \\
Q_{\phi}^{2} & =-2 \xi_{c} \xi_{a}+\xi_{a}\left(\xi_{c}-\xi_{a}\right) \sin ^{2} \eta .
\end{aligned}
$$

Notably, there is a contribution from both the $\varphi^{2}$ and $\phi^{2}$ potentials, while the $\psi^{2}$ potential is zero. This gives rise to the flow matrix

$$
\mathbf{S}=\left(\begin{array}{ccc}
0 & -\left(\boldsymbol{\Phi}_{22}^{1}+\boldsymbol{\Phi}_{22}^{2}\right) & 0 \\
\boldsymbol{\Phi}_{11}^{1}+\boldsymbol{\Phi}_{11}^{2}-\boldsymbol{\Gamma}_{31}^{2} & 0 & \boldsymbol{\Phi}_{31}^{1}+\boldsymbol{\Phi}_{31}^{2}-\boldsymbol{\Gamma}_{33}^{2} \\
0 & \boldsymbol{\Gamma}_{22}^{2} & 0
\end{array}\right)
$$

where the components of the second-order potentials are given by

$$
\begin{array}{rlrl}
\boldsymbol{\Phi}_{11}^{2} & =\frac{Q_{\phi}^{2}}{\varpi} \boldsymbol{\Phi}_{11}^{1} ; & \boldsymbol{\Phi}_{22}^{2}=\frac{Q_{\phi}^{2}}{\varpi} \boldsymbol{\Phi}_{22}^{1} ; & \boldsymbol{\Phi}_{31}^{2}=\frac{Q_{\phi}^{2}}{\varpi} \boldsymbol{\Phi}_{31}^{1} ; \\
\boldsymbol{\Gamma}_{22}^{2}=\frac{Q_{\varphi}^{2}}{\varpi} \boldsymbol{\Phi}_{22}^{1} ; & \boldsymbol{\Gamma}_{31}^{2}=\frac{Q_{\varphi}^{2}}{\varpi} \boldsymbol{\Phi}_{31}^{1} ; & \boldsymbol{\Gamma}_{33}^{2}=\frac{Q_{\varphi}^{2}}{\varpi} \boldsymbol{\Phi}_{33}^{1} .
\end{array}
$$

Considering as in the case of the ellipsoid in standard position that the vortex rotates about the $z$-axis, we can substitute this flow matrix into equation (4.29) to solve for the rotation rate about the $z$-axis. The rotation rate is again the sum of a first and a second-order term (equation 4.30), where now

$$
\Omega^{(1)}=\frac{a^{2} \xi_{a}-b^{2} \xi_{b}+\left(c^{2} \xi_{c}-a^{2} \xi_{a}\right) \sin ^{2} \eta}{a^{2}-b^{2}+\left(c^{2}-a^{2}\right) \sin ^{2} \eta}
$$

and at $\mathcal{O}\left(\epsilon^{2}\right)$,

$$
\Omega_{H}^{(2)}=-\frac{\Omega^{(1)}}{\varpi}\left(\xi_{c}\left(\xi_{a}+\xi_{b}\right)+\xi_{b}\left(\xi_{a}-\xi_{c}\right) \sin ^{2} \eta\right)-\frac{2 \xi_{b}\left(\xi_{c}-\xi_{a}\right)\left(c^{2} \xi_{c}-a^{2} \xi_{a}\right) \sin ^{2} \eta \cos ^{2} \eta}{\varpi\left(a^{2}-b^{2}+\left(c^{2}-a^{2}\right) \sin ^{2} \eta\right)} .
$$

When the tilt angle $\eta=0$ we recover the solution for the vortex in standard position as in the previous subsection. When the vortex is a tilted spheroid with $a=b$ we obtain

$$
\Omega^{(1)}=\frac{c^{2} \xi_{c}-a^{2} \xi_{a}}{c^{2}-a^{2}}
$$

for the QG solution, showing that the tilted QG spheroid rotation rate is independent of the tilt angle as was previously derived by Miyazaki, Ueno \& Shimonishi (1999). For the NQG correction at at $\mathcal{O}\left(\epsilon^{2}\right)$, we obtain

$$
\Omega_{H}^{(2)}=-\frac{\Omega^{(1)} \xi_{a}}{\varpi}\left[3\left(\xi_{c}-\xi_{a}\right) \cos ^{2} \eta+\xi_{c}+\xi_{a}\right]
$$


(a) Oblate vortex, $\sigma=0.6$
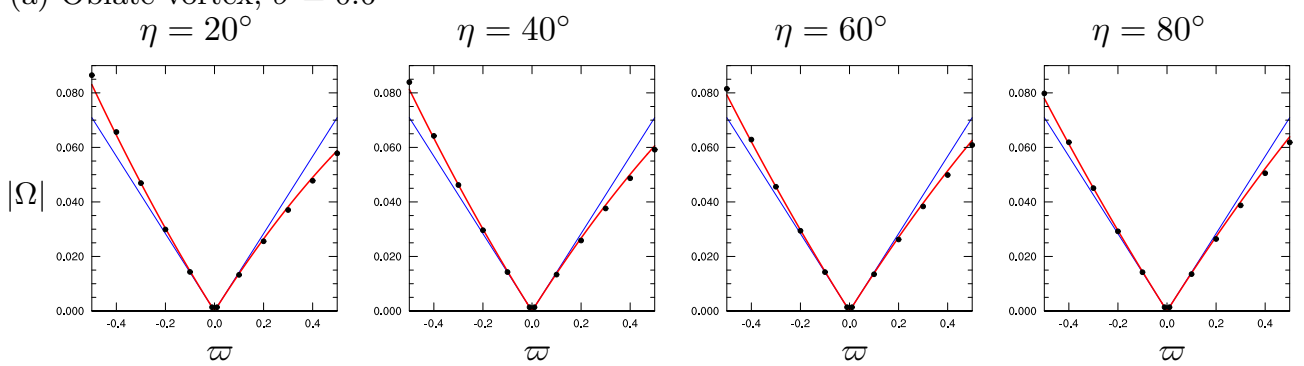

(b) Prolate vortex, $\sigma=1.6$
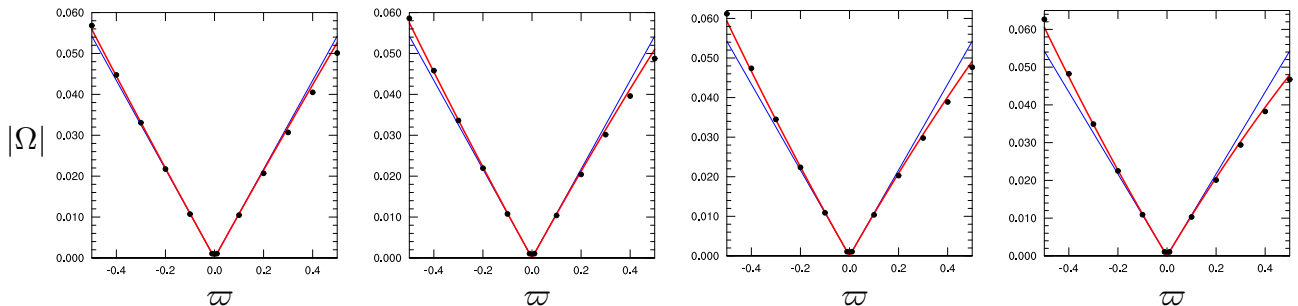

Figure 3. Plots of $|\Omega|$ versus $\varpi$ for the case of a tilted spheroid $(\delta=1)$ for four different values of the tilt angles, $\eta$, and for (a) an oblate vortex, $\sigma=0.6$, and (b) a prolate vortex, $\sigma=1.6$. The thin blue curve is the QG rotation rate, $\left|\Omega^{(1)}\right|$, the thick red curve is the correction using the homogeneous balance rotation rate $\left|\Omega^{(1)}+\Omega_{H}^{(2)}\right|$ calculated from the analytical formula, and the black points are the numerically-computed values obtained from NQG balance.

which, unlike the QG case, does depend on the tilt angle of the vortex.

In Figure 3 we now show the comparison between the analytical and numericallycomputed rotation rates for a tilted spheroid. In this case we consider two values of the vertical aspect ratio, $\sigma=0.6$ and $\sigma=1.6$ ( $\delta=1$ for a spheroid). For each of these vertical aspect ratios we consider four values of the tilt angle, $20^{\circ}, 40^{\circ}, 60^{\circ}, 80^{\circ}$. Over the full range of parameters there is excellent agreement between the analytical and numerical values, both showing the weak variation of the rotation rate with the tilt angle.

\section{Discussion and conclusions}

In this work, we have derived a balanced solution for the motion of an ellipsoidal vortex in a rotating stratified fluid at finite Rossby number. The solution extends the known quasi-geostrophic solution to one higher order in Rossby number, thereby increasing its domain of validity. The solution is obtained from the non-hydrostatic equations by imposing balance (filtering inertia-gravity waves but including ageostrophic effects). This leads to three Poisson equations, having spatially-uniform sources within the vortex for an ellipsoid of uniform potential vorticity. The sources outside of the vortex, however, are generally complicated functions of the coordinates. This leads to a difficult matching problem to find the complete analytical solution, which we are able to obtain only for an ellipsoid in standard position (with axes aligned with the coordinate axes). If on the other hand we neglect the exterior source terms, we can exploit Laplace's elegant solution of the problem (Laplace (1784)) and write down the closed analytical solution with a minimum of algebraic effort. This approximation proves to be remarkably accurate in determining the vortex dynamics even for moderate Rossby numbers, as detailed comparisons with computed solutions to the full nonlinear balance model reveal. 
In future work, it would be worthwhile investigating the stability of steadily-rotating ellipsoids at finite Rossby number. To date, our knowledge of vortex stability has mainly relied on the quasi-geostrophic approaximation (Reinaud, Dritschel \& Koudella (2003); Dritschel, Scott \& Reinaud (2005); McKiver \& Dritschel (2006)), or on full numerical simulations of the non-hydrostatic equations (Tsang \& Dritschel (2015)). The present work shows that it is possible to accurately describe the undisturbed vortex motion, analytically, to second order in the Rossby number. This may enable a corresponding analytical treatment of stability, helping to shed light on the role played by nonlinear geostrophic effects on e.g. the asymmetry exhibited by cyclonic and anti-cyclonic vortices. Moreover, it would help quantify the shift in the stability boundary in parameter space as the Rossby number increases, and in particular help identify the vortex characteristics most beneficial for stability (as done in the quasi-geostrophic case by Reinaud, Dritschel \& Koudella (2003)). Ultimately, the utility of the ellipsoidal model may be judged by comparisons of these stability characteristics with those evidently exhibited by deep, longlived, isolated three-dimensional mesoscale and sub-mesoscale vortices widely observed in oceanographic surveys (Shapiro \& Meschanov (1991), Paillet, Le Cann, Carton, Morel \& Serpette (2002), Ursella, Kovačević \& Gačić (2011), Bosse, Testor, Mortier, Prieur, Taillandier, d'Ortenzio \& Coppola (2015)).

Another opportunity to advance this work would be to extend the solutions to multiple vortices, modelled by time-varying ellipsoids, as in the quasi-geostrophic ellipsoidal model (Dritschel, Reinaud \& McKiver (2004)). This would offer a direct means to comprehensively explore the role of nonlinear ageostrophic effects on vortex interactions, all within a semi-analytical framework.

Most work concerning the ellipsoidal vortex to date has considered the simplest case of uniform PV. In this case, the solutions to the interior Poisson equations have quadratic spatial dependence, subsequently giving rise to a linear velocity field - just what is required to preserve the ellipsoidal form (McKiver \& Dritschel (2003)). Non-uniform PV was treated by Schecter \& Montgomery (2003), in the special case of a barotropic (twodimensional) Rankine vortex. They considered a vortex with a central core containing most of the vorticity and an outer region (skirt) of low vorticity decaying weakly away from the centre. They found that the rate of decay of this outer vorticity skirt can affect the stability of the vortex and its ability to resist vertical shear. A similar impact may be expected for three-dimensional, distributed ellipsoidal vortices, but the analysis appears considerably more involved. While there are analytical solutions for ellipsoids with distributed PV in special cases, e.g. for a parabolic interior PV profile matching to uniform PV outside (Chandrasekhar (1969)), the induced velocity has cubic dependence spatially and therefore does not preserve the ellipsoidal form in time. Additional approximations would be required to filter non-ellipsoidal deformations.

While the assumption of uniform PV is idealised, there is support for vortices with sharp edges having nearly discontinuous PV. In two-dimensional flows (with negligible viscosity), the shear or strain felt by a given vortex and induced by surrounding vortices readily strips away low-lying vorticity, leaving a near discontinuity at the vortex edge (cf. Dritschel (1998), figure 2, and references therein). The same process is likely to operate in realistic three-dimensional rotating, stratified flows, since the essential ingredients are the presence of shear (e.g. surrounding vortices) and negligible viscosity.

Support for this research has come from the UK Engineering and Physical Sciences Research Council (grant number EP/H001794/1). 


\section{Appendix A: Ellipsoidal coordinates and ellipsoidal harmonics}

For problems involving ellipsoidal boundaries it is often convenient to move from Cartesian coordinates $(x, y, z)$ to an ellipsoidal coordinate system $(\lambda, \mu, \nu)$ using the relations (Lyttleton (1953))

$$
\begin{aligned}
& x^{2}=\frac{\left(\lambda+a^{2}\right)\left(\mu+a^{2}\right)\left(\nu+a^{2}\right)}{\left(a^{2}-b^{2}\right)\left(a^{2}-c^{2}\right)}, \\
& y^{2}=\frac{\left(\lambda+b^{2}\right)\left(\mu+b^{2}\right)\left(\nu+b^{2}\right)}{\left(b^{2}-c^{2}\right)\left(b^{2}-a^{2}\right)}, \\
& z^{2}=\frac{\left(\lambda+c^{2}\right)\left(\mu+c^{2}\right)\left(\nu+c^{2}\right)}{\left(c^{2}-a^{2}\right)\left(c^{2}-b^{2}\right)},
\end{aligned}
$$

where the variables $\lambda, \mu$ and $\nu$ are found from the roots to the cubic equation

$$
\frac{x^{2}}{a^{2}+\chi}+\frac{y^{2}}{b^{2}+\chi}+\frac{z^{2}}{c^{2}+\chi}=1
$$

and where $\lambda>-c^{2}>\mu>-b^{2}>\nu>-a^{2}$. The $\lambda$-surfaces are ellipsoids, the $\mu$-surfaces are hyperboloids of one sheet, and the $\nu$-surfaces are hyperboloids of two sheets (Dassios (2012)). The largest root, $\lambda$, is analogous to the radial variable in spherical coordinates with each $\lambda$ representing a family of concentric ellipsoids, with $\lambda=0$ being the value on the surface of the original ellipsoid with semi-axes $a, b$ and $c$.

Working in an ellipsoidal coordinate system, a general solution to Laplace's equation for an ellipsoidal body can be written as an expansion in ellipsoidal harmonics (Hobson (1931); Lyttleton (1953); Dassios (2012))

$$
\phi_{e}=\sum_{m}^{\infty} \sum_{k}^{2 m+1} \mathbf{F}_{m}^{(k)}(\lambda) \mathbf{E}_{m}^{(k)}(\mu) \mathbf{E}_{m}^{(k)}(\nu)
$$

where $\mathbf{E}_{m}^{(k)}$ denote Lamé functions of the first kind (of order $m$ ), while $\mathbf{F}_{m}^{(k)}$ denote Lamé functions of the second kind defined by

$$
\mathbf{F}_{m}^{(k)}(\lambda)=\frac{2 m+1}{2} \mathbf{E}_{m}^{(k)}(\lambda) \int_{0}^{\infty} \frac{d t}{\left[\mathbf{E}_{m}^{(k)}(t+\lambda)\right]^{2} \sqrt{\left(t+\lambda+a^{2}\right)\left(t+\lambda+b^{2}\right)\left(t+\lambda+c^{2}\right)}}
$$

For the solution to the NQG ellipsoid we require the second-order harmonics $(m=2)$, where the Lamé functions of the first kind are given by

$$
\begin{aligned}
& \mathbf{E}_{2}^{(1)}(\lambda)=\lambda+d_{1}, \\
& \mathbf{E}_{2}^{(2)}(\lambda)=\lambda+d_{2}, \\
& \mathbf{E}_{2}^{(3)}(\lambda)=\sqrt{\left(\lambda+b^{2}\right)\left(\lambda+c^{2}\right)} \\
& \mathbf{E}_{2}^{(4)}(\lambda)=\sqrt{\left(\lambda+c^{2}\right)\left(\lambda+a^{2}\right)} \\
& \mathbf{E}_{2}^{(5)}(\lambda)=\sqrt{\left(\lambda+a^{2}\right)\left(\lambda+b^{2}\right)}
\end{aligned}
$$

where

$$
\begin{aligned}
& d_{1}=\frac{1}{3}\left[\left(a^{2}+b^{2}+c^{2}\right)+\sqrt{a^{4}+b^{4}+c^{4}-b^{2} c^{2}-c^{2} a^{2}-a^{2} b^{2}}\right] \\
& d_{2}=\frac{1}{3}\left[\left(a^{2}+b^{2}+c^{2}\right)-\sqrt{a^{4}+b^{4}+c^{4}-b^{2} c^{2}-c^{2} a^{2}-a^{2} b^{2}}\right] .
\end{aligned}
$$

The second-order harmonics of the second kind can be expressed in terms of elliptic 
integrals and are given by

$$
\begin{aligned}
& \mathbf{F}_{2}^{(1)}(\lambda)=-\frac{5}{6 \kappa_{v}}\left(\frac{\alpha^{2} \xi_{\alpha}}{d_{1}-a^{2}}+\frac{\beta^{2} \xi_{\beta}}{d_{1}-b^{2}}+\frac{\gamma^{2} \xi_{\gamma}}{d_{1}-c^{2}}\right), \\
& \mathbf{F}_{2}^{(2)}(\lambda)=-\frac{5}{6 \kappa_{v}}\left(\frac{\alpha^{2} \xi_{\alpha}}{d_{2}-a^{2}}+\frac{\beta^{2} \xi_{\beta}}{d_{2}-b^{2}}+\frac{\gamma^{2} \xi_{\gamma}}{d_{2}-c^{2}}\right), \\
& \mathbf{F}_{2}^{(3)}(\lambda)=-\frac{5 \beta \gamma\left(\xi_{\beta}-\xi_{\gamma}\right)}{3 \kappa_{v}\left(b^{2}-c^{2}\right)} \\
& \mathbf{F}_{2}^{(4)}(\lambda)=-\frac{5 \gamma \alpha\left(\xi_{\gamma}-\xi_{\alpha}\right)}{3 \kappa_{v}\left(c^{2}-a^{2}\right)} \\
& \mathbf{F}_{2}^{(5)}(\lambda)=-\frac{5 \alpha \beta\left(\xi_{\alpha}-\xi_{\beta}\right)}{3 \kappa_{v}\left(a^{2}-b^{2}\right)}
\end{aligned}
$$

where the first two formulas are obtained using formulas from Appendix G (equations (G.50) and (G.51)) of Dassios (2012), whereas the other three are based on expressions derived in Appendix A of McKiver \& Dritschel (2006). Their derivatives are given by

$$
\begin{aligned}
\frac{d \mathbf{F}_{2}^{(1)}}{d \lambda} & =-\frac{5}{6 \kappa_{v}}\left(\frac{\xi_{\alpha}}{d_{1}-a^{2}}+\frac{\xi_{\beta}}{d_{1}-b^{2}}+\frac{\xi_{\gamma}}{d_{1}-c^{2}}\right), \\
\frac{d \mathbf{F}_{2}^{(2)}}{d \lambda} & =-\frac{5}{6 \kappa_{v}}\left(\frac{\xi_{\alpha}}{d_{2}-a^{2}}+\frac{\xi_{\beta}}{d_{2}-b^{2}}+\frac{\xi_{\gamma}}{d_{2}-c^{2}}\right), \\
\frac{d \mathbf{F}_{2}^{(3)}}{d \lambda} & =-\frac{5}{2 \beta \gamma}\left[\frac{\left(\beta^{2}+\gamma^{2}\right)\left(\xi_{\beta}-\xi_{\gamma}\right)}{3 \kappa_{v}\left(b^{2}-c^{2}\right)}-\frac{1}{\alpha \beta \gamma}\right], \\
\frac{d \mathbf{F}_{2}^{(4)}}{d \lambda} & =-\frac{5}{2 \gamma \alpha}\left[\frac{\left(\gamma^{2}+\alpha^{2}\right)\left(\xi_{\gamma}-\xi_{\alpha}\right)}{3 \kappa_{v}\left(c^{2}-a^{2}\right)}-\frac{1}{\alpha \beta \gamma}\right], \\
\frac{d \mathbf{F}_{2}^{(5)}}{d \lambda} & =-\frac{5}{2 \alpha \beta}\left[\frac{\left(\alpha^{2}+\beta^{2}\right)\left(\xi_{\alpha}-\xi_{\beta}\right)}{3 \kappa_{v}\left(a^{2}-b^{2}\right)}-\frac{1}{\alpha \beta \gamma}\right],
\end{aligned}
$$

\section{Appendix B: Cartesian derivatives of ellipsoidal coordinate $\lambda$}

Beginning with the equation that defines the ellipsoidal coordinate $\lambda$ for an ellipsoid in standard position (i.e. with its axes aligned with the coordinate axes), we have

$$
\frac{x^{2}}{a^{2}+\lambda}+\frac{y^{2}}{b^{2}+\lambda}+\frac{z^{2}}{c^{2}+\lambda}=1
$$

Taking the derivative of this with respect to $x$ gives

$$
\frac{2 x}{a^{2}+\lambda}-\left[\frac{x^{2}}{\left(a^{2}+\lambda\right)^{2}}+\frac{y^{2}}{\left(b^{2}+\lambda\right)^{2}}+\frac{z^{2}}{\left(c^{2}+\lambda\right)^{2}}\right] \frac{\partial \lambda}{\partial x}=0
$$

giving us an expression for the derivative of $\lambda$ with respect to $x$,

$$
\frac{\partial \lambda}{\partial x}=\frac{2\left(a^{2}+\lambda\right)\left(b^{2}+\lambda\right)^{2}\left(c^{2}+\lambda\right)^{2} x}{\left(b^{2}+\lambda\right)^{2}\left(c^{2}+\lambda\right)^{2} x^{2}+\left(c^{2}+\lambda\right)^{2}\left(a^{2}+\lambda\right)^{2} y^{2}+\left(a^{2}+\lambda\right)^{2}\left(b^{2}+\lambda\right)^{2} z^{2}}
$$

Using the expressions $\alpha^{2} \equiv a^{2}+\lambda, \beta^{2} \equiv b^{2}+\lambda, \gamma^{2} \equiv c^{2}+\lambda$, and introducing the function $K$ which is defined as

$$
K(f, g, h)=g h x^{2}+h f y^{2}+f g z^{2},
$$


(note that $K\left(\alpha^{2}, \beta^{2}, \gamma^{2}\right)=\alpha^{2} \beta^{2} \gamma^{2}$ from Equation (B.1)) then we can rewrite the derivative as

$$
\frac{\partial \lambda}{\partial x}=\frac{2 \alpha^{2} \beta^{4} \gamma^{4} x}{K\left(\alpha^{4}, \beta^{4}, \gamma^{4}\right)} .
$$

By symmetry we have

$$
\begin{aligned}
& \frac{\partial \lambda}{\partial y}=\frac{2 \alpha^{4} \beta^{2} \gamma^{4} y}{K\left(\alpha^{4}, \beta^{4}, \gamma^{4}\right)} \\
& \frac{\partial \lambda}{\partial z}=\frac{2 \alpha^{4} \beta^{4} \gamma^{2} z}{K\left(\alpha^{4}, \beta^{4}, \gamma^{4}\right)}
\end{aligned}
$$

To obtain the second derivative we take the derivative of equation (B.2) with respect to $x$ to obtain

$$
\begin{gathered}
\frac{2}{a^{2}+\lambda}-\frac{4 x}{\left(a^{2}+\lambda\right)^{2}} \frac{\partial \lambda}{\partial x}+2\left[\frac{x^{2}}{\left(a^{2}+\lambda\right)^{3}}+\frac{y^{2}}{\left(b^{2}+\lambda\right)^{3}}+\frac{z^{2}}{\left(c^{2}+\lambda\right)^{3}}\right]\left(\frac{\partial \lambda}{\partial x}\right)^{2} \\
=\left[\frac{x^{2}}{\left(a^{2}+\lambda\right)^{2}}+\frac{y^{2}}{\left(b^{2}+\lambda\right)^{2}}+\frac{z^{2}}{\left(c^{2}+\lambda\right)^{2}}\right] \frac{\partial^{2} \lambda}{\partial x^{2}} \\
\Rightarrow \frac{K\left(\alpha^{4}, \beta^{4}, \gamma^{4}\right)}{\alpha^{4} \beta^{4} \gamma^{4}} \frac{\partial^{2} \lambda}{\partial x^{2}}=\frac{8 \beta^{2} \gamma^{2} x^{2} K\left(\alpha^{6}, \beta^{6}, \gamma^{6}\right)}{\alpha^{2}\left[K\left(\alpha^{4}, \beta^{4}, \gamma^{4}\right)\right]^{2}}-\frac{8 \beta^{4} \gamma^{4} x^{2}}{\alpha^{2} K\left(\alpha^{4}, \beta^{4}, \gamma^{4}\right)}+\frac{2}{\alpha^{2}}
\end{gathered}
$$

leading to

$$
\frac{\partial^{2} \lambda}{\partial x^{2}}=\frac{2 \alpha^{2} \beta^{2} \gamma^{2}}{K\left(\alpha^{4}, \beta^{4}, \gamma^{4}\right)}\left[\beta^{2} \gamma^{2}-\frac{4 \beta^{6} \gamma^{6} x^{2}}{K\left(\alpha^{4}, \beta^{4}, \gamma^{4}\right)}+\frac{4 \beta^{4} \gamma^{4} x^{2} K\left(\alpha^{6}, \beta^{6}, \gamma^{6}\right)}{\left[K\left(\alpha^{4}, \beta^{4}, \gamma^{4}\right)\right]^{2}}\right]
$$

Similarly we have

$$
\begin{aligned}
& \frac{\partial^{2} \lambda}{\partial y^{2}}=\frac{2 \alpha^{2} \beta^{2} \gamma^{2}}{K\left(\alpha^{4}, \beta^{4}, \gamma^{4}\right)}\left[\gamma^{2} \alpha^{2}-\frac{4 \gamma^{6} \alpha^{6} y^{2}}{K\left(\alpha^{4}, \beta^{4}, \gamma^{4}\right)}+\frac{4 \gamma^{4} \alpha^{4} y^{2} K\left(\alpha^{6}, \beta^{6}, \gamma^{6}\right)}{\left[K\left(\alpha^{4}, \beta^{4}, \gamma^{4}\right)\right]^{2}}\right] \\
& \frac{\partial^{2} \lambda}{\partial z^{2}}=\frac{2 \alpha^{2} \beta^{2} \gamma^{2}}{K\left(\alpha^{4}, \beta^{4}, \gamma^{4}\right)}\left[\alpha^{2} \beta^{2}-\frac{4 \alpha^{6} \beta^{6} z^{2}}{K\left(\alpha^{4}, \beta^{4}, \gamma^{4}\right)}+\frac{4 \alpha^{4} \beta^{4} z^{2} K\left(\alpha^{6}, \beta^{6}, \gamma^{6}\right)}{\left[K\left(\alpha^{4}, \beta^{4}, \gamma^{4}\right)\right]^{2}}\right]
\end{aligned}
$$

Using these expressions we can derive the form of the Laplacian of any arbitrary function that depends only on the ellipsoidal coordinate, i.e. $f(\lambda)$ :

$$
\nabla^{2} f(\lambda)=\frac{d^{2} f}{d \lambda^{2}}\left[\left(\frac{\partial \lambda}{\partial x}\right)^{2}+\left(\frac{\partial \lambda}{\partial y}\right)^{2}+\left(\frac{\partial \lambda}{\partial z}\right)^{2}\right]+\frac{d f}{d \lambda}\left[\frac{\partial^{2} \lambda}{\partial x^{2}}+\frac{\partial^{2} \lambda}{\partial y^{2}}+\frac{\partial^{2} \lambda}{\partial z^{2}}\right]
$$

From equations (B.5), (B.6a) and (B.6b) we have

$$
\left(\frac{\partial \lambda}{\partial x}\right)^{2}+\left(\frac{\partial \lambda}{\partial y}\right)^{2}+\left(\frac{\partial \lambda}{\partial z}\right)^{2}=\frac{4 \alpha^{4} \beta^{4} \gamma^{4}}{K\left(\alpha^{4}, \beta^{4}, \gamma^{4}\right)}
$$

whereas from equations (B.8), (B.9a) and (B.9b) we have

$$
\frac{\partial^{2} \lambda}{\partial x^{2}}+\frac{\partial^{2} \lambda}{\partial y^{2}}+\frac{\partial^{2} \lambda}{\partial z^{2}}=\frac{2 \alpha^{2} \beta^{2} \gamma^{2}}{K\left(\alpha^{4}, \beta^{4}, \gamma^{4}\right)}\left(\beta^{2} \gamma^{2}+\gamma^{2} \alpha^{2}+\alpha^{2} \beta^{2}\right) \text {. }
$$

Thus the Laplacian of $f(\lambda)$ is given by

$$
\nabla^{2} f(\lambda)=\frac{2 \alpha^{2} \beta^{2} \gamma^{2}}{K\left(\alpha^{4}, \beta^{4}, \gamma^{4}\right)}\left[2 \alpha^{2} \beta^{2} \gamma^{2} \frac{d^{2} f}{d \lambda^{2}}+\left(\beta^{2} \gamma^{2}+\gamma^{2} \alpha^{2}+\alpha^{2} \beta^{2}\right) \frac{d f}{d \lambda}\right]
$$




\section{Appendix C: Derivatives of exterior elliptic integrals}

First we consider the elliptic integral of the first kind

$$
R_{F}\left(\alpha^{2}, \beta^{2}, \gamma^{2}\right) \equiv \frac{1}{2} \int_{0}^{\infty} \frac{d t}{\sqrt{\left(t+\alpha^{2}\right)\left(t+\beta^{2}\right)\left(t+\gamma^{2}\right)}}=\frac{1}{2} \int_{0}^{\infty} \frac{d t}{\Delta_{t}}
$$

where

$$
\Delta_{t} \equiv \sqrt{\left(t+\alpha^{2}\right)\left(t+\beta^{2}\right)\left(t+\gamma^{2}\right)}
$$

Taking the derivative with respect to $\lambda$ gives

$$
\begin{array}{r}
\frac{\partial R_{F}\left(\alpha^{2}, \beta^{2}, \gamma^{2}\right)}{\partial \lambda}=-\frac{1}{2} \int_{0}^{\infty} \frac{d t}{\Delta_{t}}\left[\frac{1 / 2}{t+\alpha^{2}}+\frac{1 / 2}{t+\beta^{2}}+\frac{1 / 2}{t+\gamma^{2}}\right] \\
=-\frac{1}{6}\left[R_{D}\left(\beta^{2}, \gamma^{2}, \alpha^{2}\right)+R_{D}\left(\gamma^{2}, \alpha^{2}, \beta^{2}\right)+R_{D}\left(\alpha^{2}, \beta^{2}, \gamma^{2}\right)\right]
\end{array}
$$

Using the identity derived by Carlson (1965)

$$
R_{D}\left(\beta^{2}, \gamma^{2}, \alpha^{2}\right)+R_{D}\left(\gamma^{2}, \alpha^{2}, \beta^{2}\right)+R_{D}\left(\alpha^{2}, \beta^{2}, \gamma^{2}\right)=\frac{3}{\sqrt{\alpha^{2} \beta^{2} \gamma^{2}}}=\frac{3}{\Delta_{0}}
$$

we obtain

$$
\frac{\partial R_{F}\left(\alpha^{2}, \beta^{2}, \gamma^{2}\right)}{\partial \lambda}=-\frac{1}{2 \Delta_{0}}
$$

where

$$
\Delta_{0} \equiv \sqrt{\left(a^{2}+\lambda\right)\left(b^{2}+\lambda\right)\left(c^{2}+\lambda\right)}=\alpha \beta \gamma
$$

Next we consider the elliptic integral of the second kind

$$
R_{D}\left(\beta^{2}, \gamma^{2}, \alpha^{2}\right) \equiv \frac{3}{2} \int_{0}^{\infty} \frac{d t}{\sqrt{\left(t+\alpha^{2}\right)^{3}\left(t+\beta^{2}\right)\left(t+\gamma^{2}\right)}}=\frac{3}{2} \int_{0}^{\infty} \frac{d t}{\left(t+\alpha^{2}\right) \Delta_{t}} .
$$

Taking the derivative of this with respect to $\lambda$ gives

$$
\frac{\partial R_{D}\left(\beta^{2}, \gamma^{2}, \alpha^{2}\right)}{\partial \lambda}=-\frac{3}{2} \int_{0}^{\infty} \frac{d t}{\left(t+\alpha^{2}\right) \Delta_{t}}\left[\frac{3 / 2}{t+\alpha^{2}}+\frac{1 / 2}{t+\beta^{2}}+\frac{1 / 2}{t+\gamma^{2}}\right] .
$$

From Appendix A of McKiver \& Dritschel (2006) this expression can be written as

$$
\begin{aligned}
\frac{\partial R_{D}\left(\beta^{2}, \gamma^{2}, \alpha^{2}\right)}{\partial \lambda} & =\frac{\partial R_{D}\left(\beta^{2}, \gamma^{2}, \alpha^{2}\right)}{\partial \alpha^{2}}+\frac{\partial R_{D}\left(\gamma^{2}, \alpha^{2}, \beta^{2}\right)}{\partial \alpha^{2}}+\frac{\partial R_{D}\left(\alpha^{2}, \beta^{2}, \gamma^{2}\right)}{\partial \alpha^{2}} \\
& =\frac{\partial}{\partial \alpha^{2}}\left[R_{D}\left(\beta^{2}, \gamma^{2}, \alpha^{2}\right)+R_{D}\left(\gamma^{2}, \alpha^{2}, \beta^{2}\right)+R_{D}\left(\alpha^{2}, \beta^{2}, \gamma^{2}\right)\right]
\end{aligned}
$$

Again using the identity of Carlson (1965) we obtain

$$
\frac{\partial R_{D}\left(\beta^{2}, \gamma^{2}, \alpha^{2}\right)}{\partial \lambda}=\frac{\partial}{\partial \alpha^{2}}\left[\frac{3}{\Delta_{0}}\right]=\frac{-3}{2 \alpha^{2} \Delta_{0}}
$$

By symmetry we have also

$$
\begin{aligned}
& \frac{\partial R_{D}\left(\gamma^{2}, \alpha^{2}, \beta^{2}\right)}{\partial \lambda}=\frac{-3}{2 \beta^{2} \Delta_{0}} \\
& \frac{\partial R_{D}\left(\alpha^{2}, \beta^{2}, \gamma^{2}\right)}{\partial \lambda}=\frac{-3}{2 \gamma^{2} \Delta_{0}}
\end{aligned}
$$

From these expressions we can calculate the source terms for the exterior field in the 
NQG equations. In particular the term

$$
\frac{\partial \xi_{\alpha}}{\partial \lambda} x^{2}+\frac{\partial \xi_{\beta}}{\partial \lambda} y^{2}+\frac{\partial \xi_{\gamma}}{\partial \lambda} z^{2}=-\frac{3 \kappa_{v}}{2 \Delta_{0}}
$$

obtained using equations (B.1), (C.10) and (C.11). Thus applying this equation along with the derivative of the elliptic integral of the first kind, equation (C.5), we find that

$$
\frac{\partial \xi_{\alpha}}{\partial \lambda} x^{2}+\frac{\partial \xi_{\beta}}{\partial \lambda} y^{2}+\frac{\partial \xi_{\gamma}}{\partial \lambda} z^{2}-3 \frac{\partial R_{F}}{\partial \lambda}=0
$$

\section{Appendix D: Derivation of outer source terms of the NQG equations}

As shown in Section 2 the source terms appearing on the right-hand sides of the NQG equations are

$$
\begin{aligned}
Q_{\varphi}^{2} & =\frac{2}{\chi}\left(\phi_{y y}^{1} \phi_{z x}^{1}-\phi_{y z}^{1} \phi_{x y}^{1}\right), \\
Q_{\psi}^{2} & =\frac{2}{\chi}\left(\phi_{x x}^{1} \phi_{y z}^{1}-\phi_{z x}^{1} \phi_{x y}^{1}\right), \\
Q_{\phi}^{2} & =\left|\nabla \phi_{z}^{1}\right|^{2}-\left(\nabla^{2} \phi^{1}\right) \phi_{z z}^{1}=\left(\phi_{z x}^{1}\right)^{2}+\left(\phi_{y z}^{1}\right)^{2}+\left(\phi_{z z}^{1}\right)^{2} .
\end{aligned}
$$

If we consider an ellipsoid in standard position, i.e. with its axes aligned along the Cartesian axes, then the outer solution at first order is

$$
\phi_{E}^{1}=\frac{1}{2}\left(\xi_{\alpha} x^{2}+\xi_{\beta} y^{2}+\xi_{\gamma} z^{2}\right)-\frac{3}{2} \kappa_{v} R_{F}\left(\alpha^{2}, \beta^{2}, \gamma^{2}\right)
$$

Taking the derivative with respect to $x$ we obtain

$$
\frac{\partial \phi_{E}^{1}}{\partial x}=\xi_{\alpha} x+\frac{1}{2} \frac{\partial \lambda}{\partial x}\left[\frac{\partial \xi_{\alpha}}{\partial \lambda} x^{2}+\frac{\partial \xi_{\beta}}{\partial \lambda} y^{2}+\frac{\partial \xi_{\gamma}}{\partial \lambda} z^{2}-3 \frac{\partial R_{F}}{\partial \lambda}\right]
$$

As shown in Appendix $\mathrm{C}$ the second term vanishes giving

$$
\frac{\partial \phi_{E}^{1}}{\partial x}=\xi_{\alpha} x .
$$

Similarly we have

$$
\begin{aligned}
\frac{\partial \phi_{E}^{1}}{\partial y} & =\xi_{\beta} y, \\
\frac{\partial \phi_{E}^{1}}{\partial z} & =\xi_{\gamma} z .
\end{aligned}
$$

Taking the derivative of equation (D.4) with respect to $x$ gives

$$
\frac{\partial^{2} \phi_{E}^{1}}{\partial x^{2}}=\xi_{\alpha}+\frac{\partial \xi_{\alpha}}{\partial \lambda} \frac{\partial \lambda}{\partial x} x=\xi_{\alpha}-\frac{3 \kappa_{v} \beta^{4} \gamma^{4} x^{2}}{\Delta_{0} K\left(\alpha^{4}, \beta^{4}, \gamma^{4}\right)}
$$

where we have used the formulas derived in Appendix B and Appendix C. Similarly by symmetry we have

$$
\begin{aligned}
\frac{\partial^{2} \phi_{E}^{1}}{\partial y^{2}} & =\xi_{\beta}-\frac{3 \kappa_{v} \gamma^{4} \alpha^{4} y^{2}}{\Delta_{0} K\left(\alpha^{4}, \beta^{4}, \gamma^{4}\right)}, \\
\frac{\partial^{2} \phi_{E}^{1}}{\partial z^{2}} & =\xi_{\gamma}-\frac{3 \kappa_{v} \alpha^{4} \beta^{4} z^{2}}{\Delta_{0} K\left(\alpha^{4}, \beta^{4}, \gamma^{4}\right)} .
\end{aligned}
$$


Similarly the cross derivatives needed in the source terms can be calculated as

$$
\begin{aligned}
& \frac{\partial^{2} \phi_{E}^{1}}{\partial x \partial y}=-\frac{3 \kappa_{v} \alpha^{2} \beta^{2} \gamma^{4} x y}{\Delta_{0} K\left(\alpha^{4}, \beta^{4}, \gamma^{4}\right)}, \\
& \frac{\partial^{2} \phi_{E}^{1}}{\partial z \partial x}=-\frac{3 \kappa_{v} \alpha^{2} \beta^{4} \gamma^{2} z x}{\Delta_{0} K\left(\alpha^{4}, \beta^{4}, \gamma^{4}\right)}, \\
& \frac{\partial^{2} \phi_{E}^{1}}{\partial y \partial z}=-\frac{3 \kappa_{v} \alpha^{4} \beta^{2} \gamma^{2} y z}{\Delta_{0} K\left(\alpha^{4}, \beta^{4}, \gamma^{4}\right)} .
\end{aligned}
$$

Using these we obtain the source terms

$$
\begin{aligned}
Q_{\varphi}^{2} & =-\left(\frac{6 \kappa_{v} \alpha^{2} \beta^{4} \gamma^{2} \xi_{\beta}}{\chi \Delta_{0} K\left(\alpha^{4}, \beta^{4}, \gamma^{4}\right)}\right) z x \\
Q_{\psi}^{2} & =-\left(\frac{6 \kappa_{v} \alpha^{4} \beta^{2} \gamma^{2} \xi_{\alpha}}{\chi \Delta_{0} K\left(\alpha^{4}, \beta^{4}, \gamma^{4}\right)}\right) y z \\
Q_{\phi}^{2} & =\xi_{\gamma}^{2}+\left[\frac{3 \kappa_{v} \alpha^{4} \beta^{4}}{\Delta_{0} K\left(\alpha^{4}, \beta^{4}, \gamma^{4}\right)}\left(\frac{3 \kappa_{v}}{\Delta_{0}}-2 \xi_{\gamma}\right)\right] z^{2} .
\end{aligned}
$$

\section{REFERENCES}

Baer, F. \& Tribbia, J. J. 1977 On complete filtering of gravity modes through nonlinear initialization. Mon. Wea. Rev. 105, 1536-1539.

BokHove, O. 1997 Slaving principles, balanced dynamics, and the Hydrostatic Boussinesq Equations. J. Atmos. Sci. 54, 1662-1674.

Bosse, A., Testor, P., Mortier, L., Prieur, L., Taillandier, V., D’Ortenzio, F. F. \& Coppola, L. 2015 Spreading of Levantine Intermediate Waters by submesoscale coherent vortices in the northwestern Mediterranean Sea as observed with gliders. J. Geophys. Res. Oceans 120, 1599-1622.

Carlson, B. C. 1965 On computing elliptic integrals and functions. J. Math. and Phys. 44, $35-51$.

Chandrasekhar, S. 1969 Ellipsoidal Figures of Equilibrium. Dover, New York, pp. 255.

Charney, J. G. 1948 On the scale of atmospheric motions. Geofys. Publ. 17(2), 3-17.

D'Asaro, E. 1988 Observations of small eddies in the Beaufort Sea. J. Geophys. Res. 93, 6669-6684.

DAssios, G. 2012 Ellipsoidal Harmonics: Theory and Applications. Cambridge University Press, pp. 474.

Dritschel, D. G. 1990 The stability of elliptical vortices in an external straining flow. J. Fluid Mech. 210, 223-261, 1990.

Dritschel, D. G. 1998 On the persistence of non-axisymmetric vortices in inviscid twodimensional flows. J. Fluid Mech. 371, 141-155, 1998.

Dritschel, D. G. \& Viúdez, Á. 2003 A balanced approach to modelling rotating stably stratified geophysical flows. J. Fluid Mech. 488, 123-150.

Dritschel, D. G, Reinaud, J. N., McKiver, W. J. 2004 The quasi-geostrophic ellipsoidal vortex model. J. Fluid Mech., 505, 201-223.

Dritschel, D. G., Scott, R. K. \& Reinaud, J. N. 2005 The stability of quasi-geostrophic ellipsoidal vortices. J. Fluid Mech. 536, 401-421.

Dritschel, D. G. \& Viúdez, Á. 2007 The persistence of balance in geophysical flows. J. Fluid Mech. 570, 365-383.

Dritschel, D. G. \& McKiver W. J. 2015 Effect of Prandtl's ratio on balance in geophysical turbulence J. Fluid Mech. 777, 569-590.

Ford, R., McIntyre, M. E. \& Norton, W. A. 2000 Balance and the slow quasimanifold: Some explicit results. J. Atmos. Sci. 57, 1236-1254.

Hashimoto, H., Shimonishi, T. \& Miyazaki, T. 1999 Quasigeostrophic ellipsoidal vortices in a two-dimensional strain field. J. Phys. Soc Jpn. 68(12), 3863-3880. 
Hobson, E. W. 1931 Theory of Spherical and Ellipsoidal Harmonics. Cambridge University Press, pp. 514.

KIDA, S. 1981 Motion of an elliptical vortex in a uniform shear flow. J. Phys. Soc Jpn. 50, 3517-3520.

KirchHoff, G. 1876 Vorlesungen ??ber matematische Physic: Mechanik Teubner, Leipzig.

Koshel, K. V., Ryzhov, E. A. \& Zhmur, V. V. 2013 Diffusion-affected passive scalar transport in an ellipsoidal vortex in a shear flow. Nonlin. Processes Geophys., 20, 437-444.

Koshel, K. V., Ryzhov, E. A. \& Zhmur, V. V. 2015 Effect of the vertical component of diffusion on passive scalar transport in an isolated vortex model. Phys. Rev. E, 92, 053021.

Lane, T. P., Doyle, J. D., Plougonven, R., Shapiro, M. A. \& Sharman, R. D. 2004 Observations and numerical simulations of inertia-gravity waves and shearing instabilities in the vicinity of a jet stream. J. Atmos. Sci. 61, 2692-2706.

Laplace, P. S. 1784 Théorie du Mouvement et de la Figure Elliptique des Planetès. Imprimerie de Ph.-D. Pierres, Paris, 153 pp.

LEITH, C. E. 1980 Nonlinear normal mode initialization and quasi-geostrophic theory. J. Atmos. Sci. 37, 958-968.

Love, A. E. H. 1893 On the stability of certain vortex motions. Proc. Lond. Math. Soc. 25, $18-42$.

Lyttleton, R. A. 1953 The stability of rotating liquid masses Cambridge University, Paris, $160 \mathrm{pp}$.

McKiver, W. J. \& Dritschel, D. G. 2003 The motion of a fluid ellipsoid in a general linear background flow. J. Fluid Mech. 474, 147-173.

McKiver, W. J. \& Dritschel, D. G. 2006 The stability of a quasi-geostrophic ellipsoidal vortex in a background shear flow J. Fluid Mech. 560, 1-17.

McKiver, W. J. \& Dritschel, D. G. 2008 Balance in non-hydrostatic rotating stratified turbulence. J. Fluid Mech. 596, 201-219.

MCKiver, W. J. 2015 The ellipsoidal vortex: A novel approach to geophysical turbulence. Adv. Mathematical Physics 2015, 613683.

Meacham, S. P. 1992 Quasigeostrophic, ellipsoidal vortices in a stratified fluid. Dyn. Atmos. Oceans 16, 189-223.

Meacham, S. P., Pankratov, K. K., Shchepetkin, A. F. \& Zhmur, V. V. 1994 The interaction of ellipsoidal vortices with background shear flows in a stratified fluid. Dyn. Atmos. Oceans 21, 167-212.

Miyazaki, T., Ueno, K. \& Shimonishi, T. 1999 Quasigeostrophic tilted spheroidal vortices. J. Phys. Soc Jpn. 68(8), 2592-2601.

Miyazaki, T., Furuichi Y. \& TAKahashi N. 2001 Quasigeostrophic ellipsoidal vortex model. J. Phys. Soc Jpn. 70(7), 1942-1953.

Mohebalhojeh, A. R., \& Dritschel, D. G. 2001 Hierarchies of balance conditions for the $f$-plane shallow water equations. J. Atmos. Sci. 58(16), 2411-2426.

Mohebalhojeh, A. R. 2002 On shallow-water potential-vorticity inversion by Rossby-number expansions. Quart. J. Roy. Meteor. Soc. 128, 679-694.

Muraki, D. J., Snyder, C. \& Rotunno, R. 1999 The next-order corrections to quasigeostrophic theory. J. Atmos. Sci. 56, 1547-1560.

Paillet, J., Le Cann, B., Carton, X., Morel, Y. \& Serpette, A. 2002 Dynamics and evolution of a Northern Meddy. J. Phys. Oceanogr. 32, 55-79.

Reinaud, J., Dritschel, D. G., \& Koudella, C. R. 2003 The shape of vortices in quasigeostrophic turbulence. J. Fluid Mech. 474, 175-191.

Schecter, D. A. \& Montgomery, M. T. 2003 On the symmetrization rate of an intense geophysical vortex. Dyn. Atmos. Oceans 37, 55-88.

Schultz-Tokos, K. \& Rossby, T. 1991 Kinematics and dynamics of a Mediterranean salt lens. J. Phys. Oceanogr. 21, 879-892.

Shapiro, G. I. \& Meschanov, S. L. 1991 Distribution and spreading of Red Sea Water and salt lens formation in the northwest Indian Ocean Deep Sea Res. 38(1), 21-34.

Tsang, Y-K. \& Dritschel, D. G. 2015 Ellipsoidal vortices in rotating stratified fluids: beyond the quasi-geostrophic approximation. J. Fluid Mech. 762, 196-231.

Ursella, L., KovaC̆ević, V. \& GaC̆IĆ, M. 2011 Footprints of mesoscale eddy passages in the Strait of Otranto (Adriatic Sea). J. Geophys. Res. 116, C04005. 
VALLIS, G. K. 1996 Potential vorticity inversion and balanced equations of motion for rotating and stratified flows. Q. J. R. Met. Soc. 122, 291-322.

VANnESte, J. \& YAVNeH, I. 2004 Exponentially small inertia-gravity waves and the breakdown of quasi-geostrophic balance. J. Atmos. Sci. 61, 211-223.

Viúdez, Á. \& Dritschel, D. G. 2004 Optimal potential vorticity balance of geophysical flows. J. Fluid Mech. 521, 343-352.

Viúdez, Á. \& Dritschel, D. G. 2006 Spontaneous generation of inertia-gravity wave packets by balanced geophysical flows. J. Fluid Mech. 553, 107-117.

Waite, M. L. \& BARTello, P. 2006 The transition from geostrophic to stratified turbulence. J. Fluid Mech. 568, 89-108.

Warn, T, Bokhove, O., Shepherd, T. G. \& Vallis, G. K. 1995 Rossby number expansions, slaving principles, and balance dynamics. Quart. J. Roy. Meteor. Soc. 121, 723-739. 